\title{
Artificial Anisotropy in Circular Photonic Crystals and Applications
}

\author{
Alessandro Massaro, Roberto Cingolani, Massimo De Vittorio, and Adriana Passaseo
}

\begin{abstract}
In this paper, we analyze the birefringence effect in circular photonic crystals (CphCs). The studied $\mathrm{CphCs}$ are dielectric rings (DRs) and photonic crystals with cylindrical air holes arranged in circular patterns. The dielectric concentric circular patterns admit two preferred propagation directions defined by an extraordinary and an ordinary refractive index, representing two electric field polarizations. These electric fields are diffracted inside the crystal or are localized in a central microcavity region. We prove the induced artificial anisotropy in DRs through the geometrical equivalence with the corresponding thin-film multilayer structure. The equivalence is obtained through the geometrical synthesis of the wavefront propagation inside the artificial anisotropic structure. As applications, we analyze a $\mathrm{Si} / \mathrm{SiO}_{2}$ DR Bragg reflector and a GaAs CphC microcavity resonator. The Bragg theory is validated by numerical time-domain approaches that are well suited to solve scattering problems. The microcavity resonance analysis and the $Q$-factor evaluation are performed by the finite-element method modeling.
\end{abstract}

Index Terms-Bragg scattering, circular photonic crystal (CphC), form birefringence, microcavity resonator, optical rings.

\section{INTRODUCTION}

$\mathbf{T}$ HE BIREFRINGENT properties of crystals may be explained in terms of the anisotropic electrical properties of molecules of which the crystals are composed. Birefringence may, however, arise from anisotropy on a scale much larger than molecular, namely when there is an arrangement of similar particles of optically isotropic material whose size is large if compared with the dimensions of molecules, but small if compared with the wavelength of light. We then speak of form birefringence. The form birefringence (induced artificial anisotropy) performs the important function of separating an incident beam into two orthogonally polarized outgoing beams. Such a function can be accomplished by isotropic periodic dielectric structures that can split an incident beam into two preferred directions, as in uniaxial crystals [1]-[10], in Bragg reflectors, and in polarization splitters. In particular, it is very interesting to note the application of the artificial anisotropy in the circular geometries of Fig. 1(a) and (b). In fact, the circular dielec-

Manuscript received November 24, 2008; revised May 5, 2009. First published August 7, 2009; current version published March 10, 2010. The review of this paper was arranged by Associate Editor S. Krishna.

A. Massaro and R. Cingolani are with IIT (Italian Institute of Technology), Center of Bio-Molecular Nanotechnology, Arnesano, 73100, Lecce (e-mail: alessandro.massaro@iit.it).

M. De Vittorio and A. Passaseo are with the National Nanotechnology Laboratory of CNR INFM, Lecce 73100, Italy.

Color versions of one or more of the figures in this paper are available online at http://ieeexplore.ieee.org.

Digital Object Identifier 10.1109/TNANO.2009.2028690 tric patterns [6], [10]-[14], arranged in ring configuration, are suitable for practical applications in which whispering gallery modes (WGMs) [11] (modes with high quality factor) are requested. This configuration is also suitable for devices in which the localized bound states, supported by cavity defects, generate different wavelength emissions associated to degenerate modes correlated to the polarization [6], [15]. The presence of degenerate modes, such as ordinary and extraordinary modes, can also be explained by the form birefringence effect induced in the circular photonic crystal (CphC) [6] of Fig. 1(a) and (b). From optical theory [2], [5], the form birefringence is analyzed by considering the idealized case of a regular assembly of particles that have the form of thin parallel plates. This idealized case can be extended to dielectric structures with circular geometries such as dielectric rings (DRs) and $\mathrm{CPhC}$ devices. The analogy between the DR and the thin-film parallel plates is obtained by the geometrical rotation of the equivalent dielectric multilayer structure around the symmetry $y$-axis [reported in Fig. 1(a)] of the circular device [6]. We observe that the DR geometries represent the extended case of $\mathrm{CPhC}$ device of Fig. 1(b): in fact, the concentric air DRs are the extended case of infinite air cylinders arranged in a circular pattern [6] [see cylindrical air holes reported in Fig. 1(a)]. By considering appropriate physical conditions and combinations of materials with different dimensions, these structures behave as Bragg reflectors and microcavity resonators, in which two modes (ordinary mode and extraordinary mode) are propagated along the structure or are localized in a central cavity region. As reported in Fig. 1(a), the two modes are associated to an ordinary electric field $\mathbf{E}_{o}$ (tangential to the rings) and an extraordinary electric field $\mathbf{E}_{e}$ (radial component). The ordinary electric field component will be characterized by an ordinary refractive index $n_{o}$ and the extraordinary electric field component will be characterized by an extraordinary refractive index $n_{e}$. Both the refractive indexes define the refractive index ellipse associated to the artificial anisotropic structure.

We analyze the following points in this paper.

1) We study the form birefringence of a DR structure that behaves as a corresponding thin-film multilayer structure characterized by an optical axis orientation and by a planewave incidence angle. The equivalence with the thin-film multilayer structure is obtained through the geometrical synthesis of the refractive index ellipse associated to the propagating wavefront.

2) As first application, we apply the Bragg condition to $\mathrm{Si} / \mathrm{SiO}_{2}$ DR Bragg reflectors. The Bragg diffraction theory, including conservation of momentum vectors, allows to calculate the Bragg angles of the ordinary and 
(a)

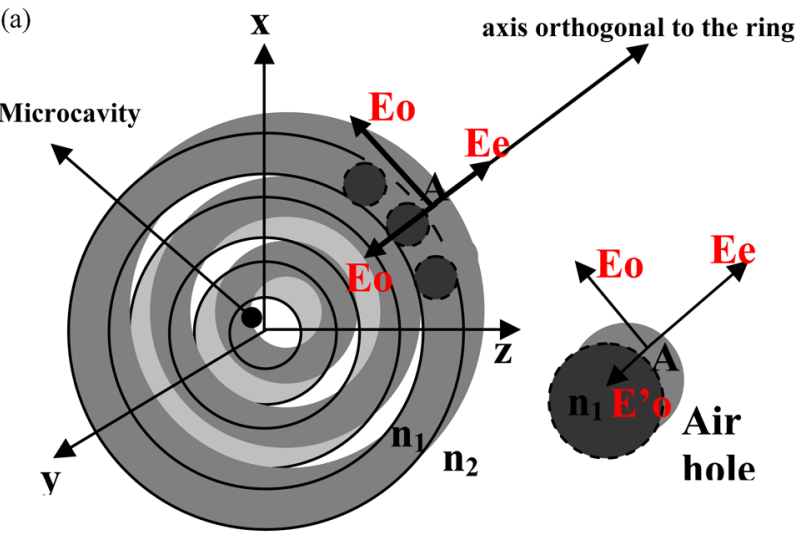

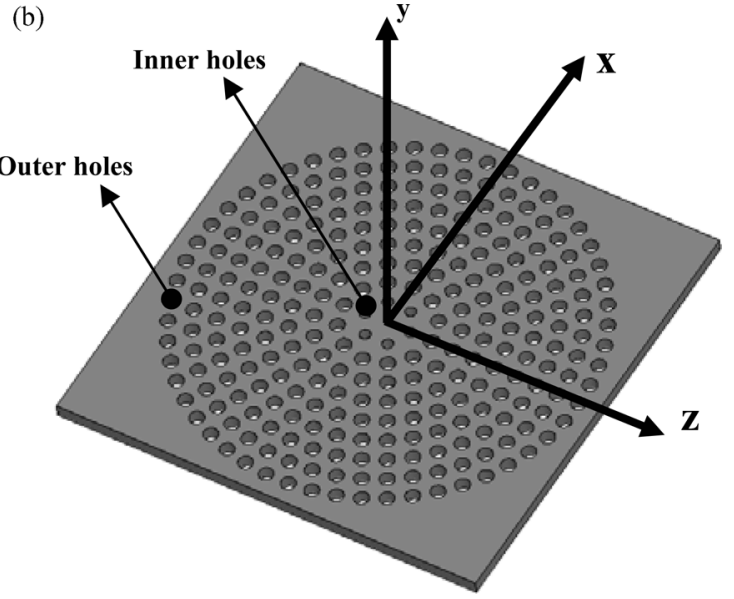

Fig. 1. (a) Air DRs $\left(n_{1}=1\right)$ in dielectric material with refractive index $n_{2}$. The concentric air rings $\left(n_{1}=1\right)$ are the extended case of infinite air cylinders arranged in a circular pattern. (b) $\mathrm{CphC}$ with central microcavity.

(a)

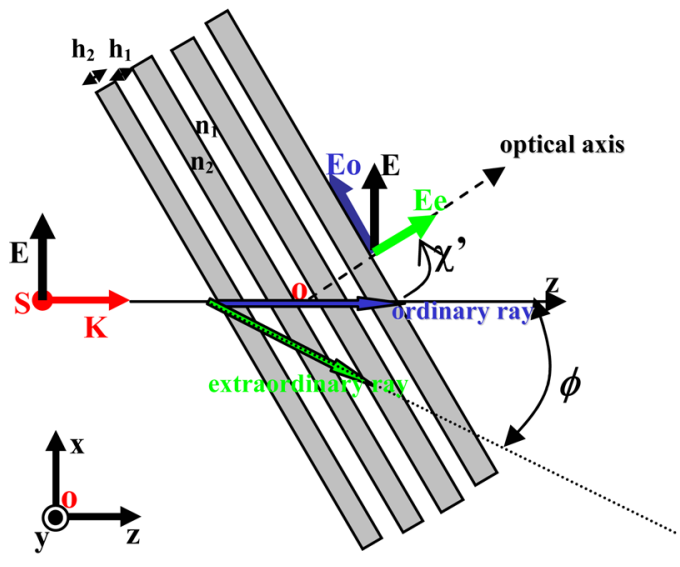

(b)

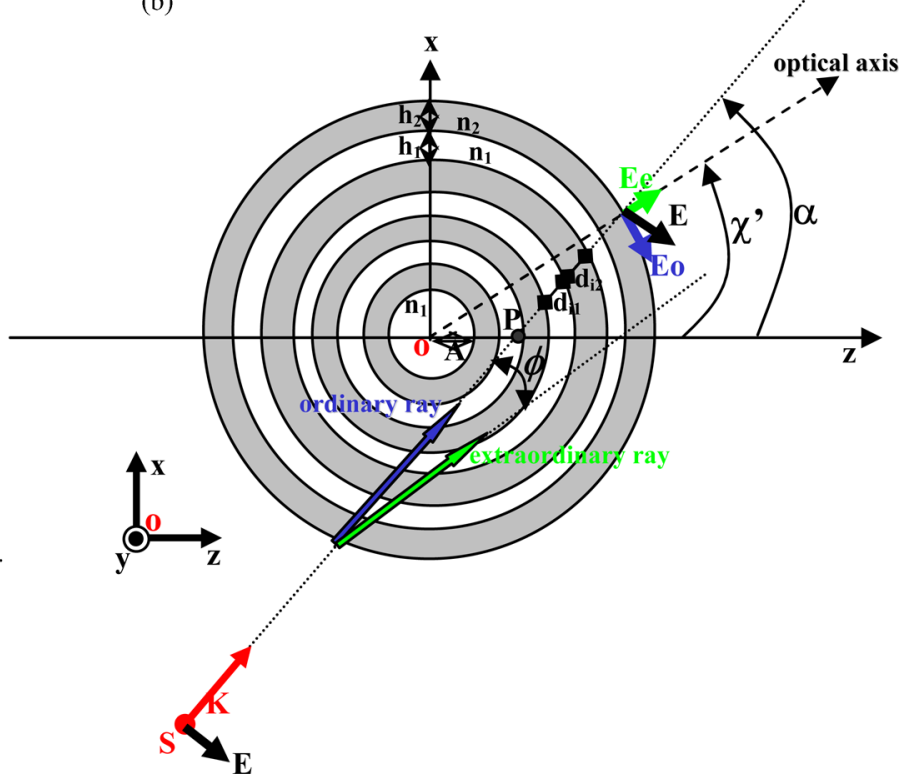

Fig. 2. (a) Birefringence form in thin parallel dielectric plates. (b) Equivalent dielectric concentric rings structure with central cavity.

extraordinary rays for different optical wavelengths and ring thicknesses. The calculated Bragg angles, which display maximum intensity as a result of constructive interference, are verified through the 2-D finite-difference time-domain (FDTD) modeling and by the Hertzian potential formulation (HPF) [16], which is typically used to solve scattering and wave propagation problems.

3) As second application, we analyze the GaAs CPhCs microcavity resonator of Fig. 1(b). In particular, we study the mode degeneracy observed in the central microcavity region. The numerical results are compared with the equivalent DR structure. The frequency responses, the $Q$-factor, and the electric field displacement are performed by a finite-element method (FEM) tool optimized for this kind of devices [17].

\section{FORM BIREFRINGENCE AND WAVE PROPAGATION OF THE CONCENTRIC DIELECTRIC OPTICAL RINGS: EQUIVALENT RING CONFIGURATION}

Dielectric periodic multilayer structures (the dielectric multilayer structure is characterized by two dielectric layers of thicknesses $h_{1}$ and $h_{2}$, with refractive indexes $n_{1}$ and $n_{2}$, respectively, which alternate periodically) select, as in negative uniaxial crystals [5], two modes (ordinary and extraordinary modes) that propagate in the structure along two different directions. The two modes are associated to an extraordinary and an ordinary field component: as reported in Fig. 2(a), the extraordinary field component $\mathbf{E}_{e}$ is parallel to the optical axis and the ordinary field $\mathbf{E}_{o}$ is orthogonal to $\mathbf{E}_{e}$; moreover, the direction of the wave vector $\mathbf{K}$ is assumed along the $z$-direction and the optical axis is defined orthogonal to the dielectric layers. 
(a)

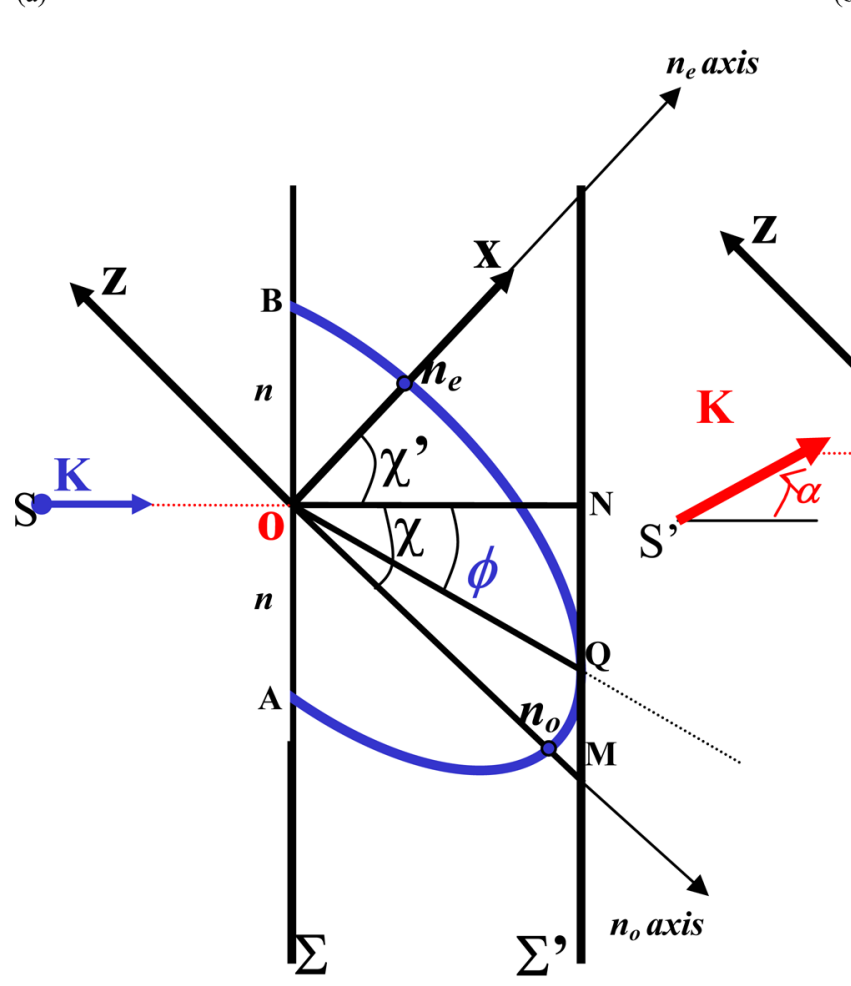

(b)

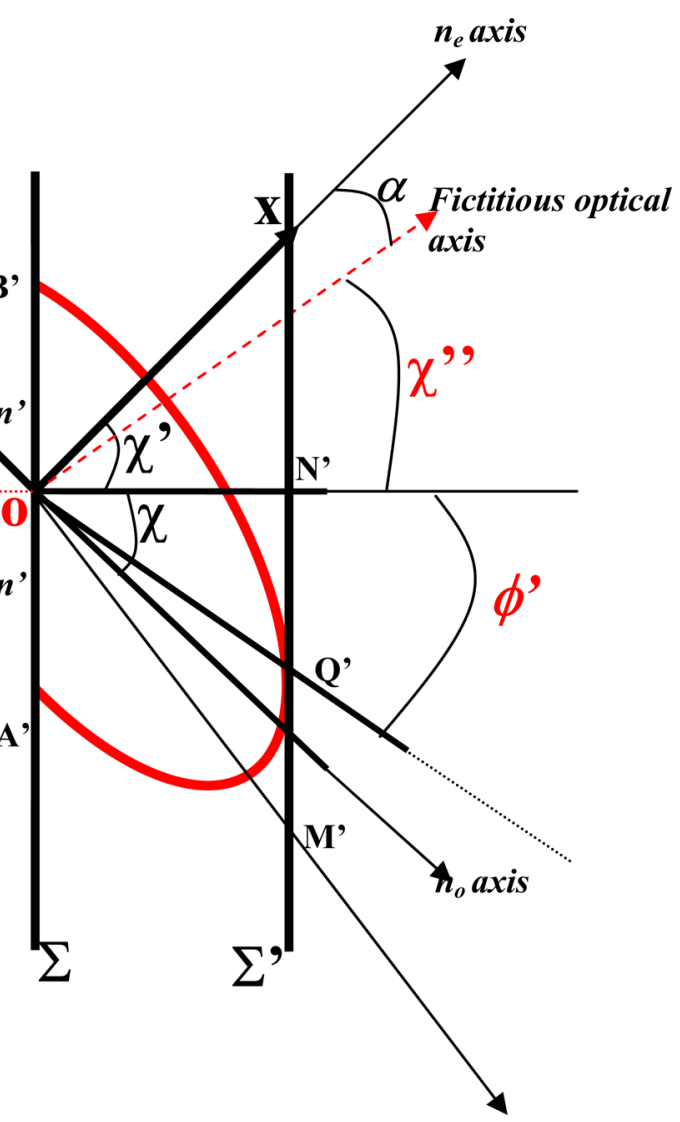

Fig. 3. (a) Wavefront and refractive index ellipse related to a thin-film multilayer structure at a particular propagation time. The $n_{e}$-axis is defined along the $x$-direction and the $n_{o}$-axis is defined along the $z$-direction. (b) Wavefront and refractive index ellipse related to the corresponding DR structure at a particular propagation time and incidence angle $\alpha$; the value $n^{\prime}=n_{e}\left(\chi^{\prime}\right)=\mathbf{O B} \mathbf{B}^{\prime}$ changes with the direction of $\mathbf{K}$. The direction of $\mathbf{K}$ is in the $x-z$ plane of the periodic structure.

Concerning a dielectric multilayer structure with circular geometry, we define [see Fig. 2(b)] the corresponding electric field configuration: in this case, the field component $\mathbf{E}_{e}$ is radial (orthogonal to the ring) and the component $\mathbf{E}_{o}$ is tangential to the ring.

The analogy of the refractive index ellipse, between the structures reported in Fig. 2(a) and (b), is analyzed for application points $\mathbf{S}$ of the $\mathbf{K}$ vector not centered to the origin of the $x-z$ axis [6]: this hypothesis takes into account that for each angle $\alpha$, the propagation direction does not intersect a periodic structure [see Fig. 2(b)] by losing the circular symmetry that is obtained by applying the $\mathbf{K}$ vector at the center $\mathrm{O}$ of the $x-z$ axis (in this case, the propagation direction will cross a periodic circular multilayer structure and the refractive index ellipse will degenerate into a circle with $n_{e}=n_{o}$ ). By rotating the equivalent multilayer structure of Fig. 2(a) around the $y$-axis, the wave vector will be characterized by different wavefront propagations ( $\Sigma$ and $\Sigma^{\prime}$ are the wavefronts at different times): for a rotation of the optical axis, the wavefront $\Sigma^{\prime}$ will be shifted and the refractive index ellipse [5], [6] will rotate by changing the value of the extraordinary refractive index [6]. Therefore, the equivalence between the ellipses of Fig. 2(a) and (b) is obtained by fixing the wave vector $\mathbf{K}$ of the thin-film multilayer structure, and by fixing a specific angle $\alpha$ and a position $\mathrm{S}$ of the corresponding $\mathbf{K}$ vector of the ring structure. The equivalence of the two structures is proved through the superposition of the refractive index ellipses of Fig. 3(a) and (b), where the split angle $\phi$ is reported, which defines the ordinary and the extraordinary propagating rays. The relationship between the split angle $\phi$ (split angle related to the ordinary and extraordinary fields in the periodic plane) and the incidence angle $\alpha$ is estimated by means of Huygens principle, starting from the ordinary and extraordinary wavefronts [2], [3], [5]. The ordinary and the extraordinary wavefronts can be superimposed to the refractive index ellipse for particular $\tau$ values, where $\tau$ is the propagation time of the wavefront in the periodic structure. In particular, for normal plane-wave incidence, $\Sigma^{\prime}$ is the envelope at the time $t+\tau$ of wavelets emitted from the various points of the wavefront $\Sigma$ at the time $t$. We see from Fig. 3(a) that $\mathbf{O N} / \tau$ is the velocity of the wave and $\mathbf{O Q} / \tau$ is the propagation velocity of the ray. Under the hypothesis of a wavelet centered in the origin $\mathbf{O}$ of the $x-z$ axis, we obtain the following relationships: $\chi=\pi / 2-\chi^{\prime}$, $d \chi^{\prime}=-d \chi, x=-n \cos \chi^{\prime}$, and $z=-n \sin \chi^{\prime}$, where $n=n_{e}\left(\chi^{\prime}\right)=\mathbf{O B}$ is the refractive index of the $\Sigma$ envelope. The ellipse equation in the $x-z$ plane for an optical axis orientation $\chi^{\prime}$ is

$$
\frac{\cos ^{2} \chi^{\prime}}{n_{e}^{2}}+\frac{\sin ^{2} \chi^{\prime}}{n_{o}^{2}}=\frac{1}{n^{2}}
$$


(a)

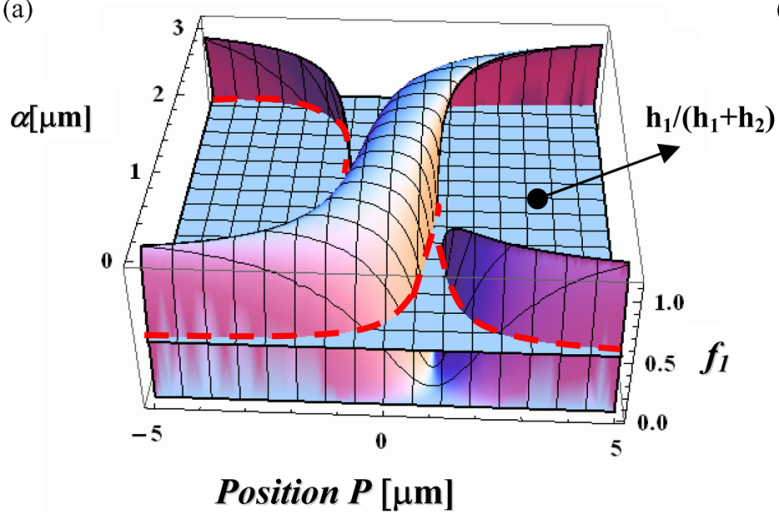

(c)

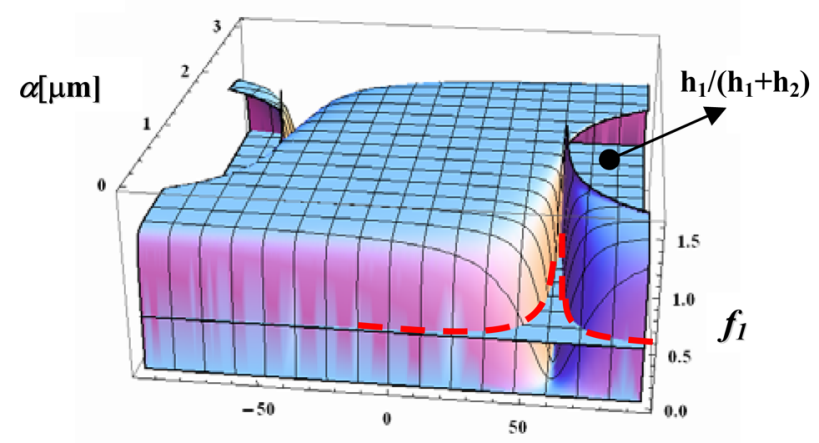

Position P $[\mu \mathrm{m}]$ (b)

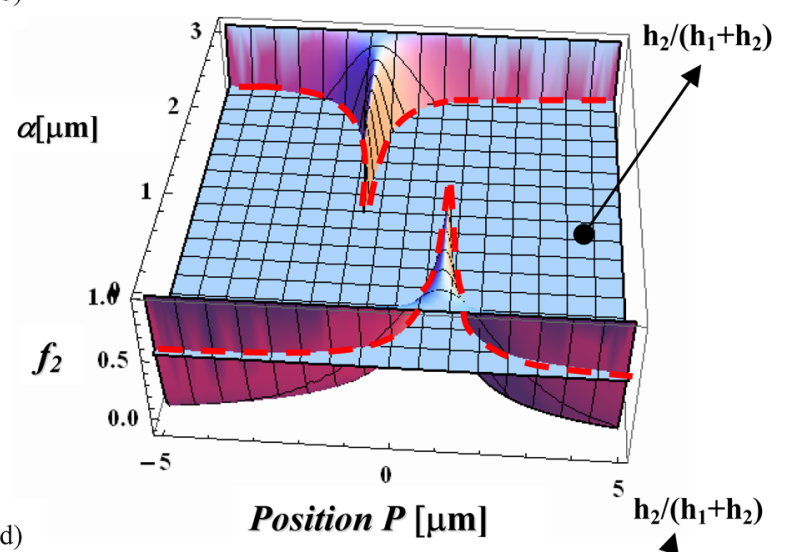

(d)

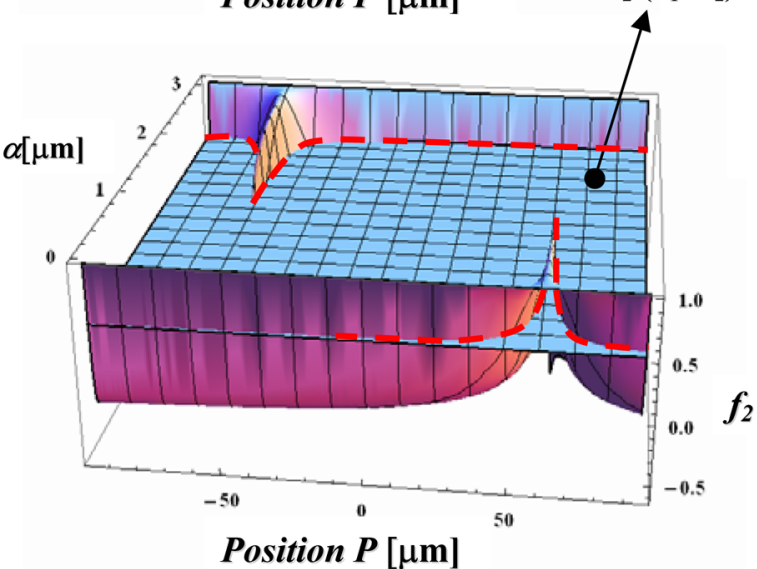

Fig. 4. Calculated filling factors (a) $f_{1}$ and (b) $f_{2}$ versus $\alpha$ and OP for a ring dielectric structure with $A=1 \mu \mathrm{m}$, and $h_{1}=h_{2}=0.01 \mu \mathrm{m}$. Calculated filling factors (c) $f_{1}$ and (d) $f_{2}$ versus $\alpha$ and OP for a ring dielectric structure with $A=60 \mu \mathrm{m}$, and $h_{1}=h_{2}=0.25 \mu \mathrm{m}$.

By differentiation, we get [3], [5]

$$
\frac{d n}{d \chi^{\prime}}=n^{3}\left(\frac{1}{n_{e}^{2}}-\frac{1}{n_{o}^{2}}\right) \sin \chi^{\prime} \cos \chi^{\prime} .
$$

The relation between the angle $\phi$ and the variation of the refractive index $n$ with respect to the angle $\chi^{\prime}$ is as follows [3], [5]:

$$
\tan \phi=-\frac{1}{n} \frac{d n}{d \chi^{\prime}}=-n^{2}\left(\frac{1}{n_{e}^{2}}-\frac{1}{n_{o}^{2}}\right) \sin \chi^{\prime} \cos \chi^{\prime}
$$

where

$$
n^{2}=\frac{n_{e}^{2} n_{o}^{2}}{n_{o}^{2} \sin ^{2} \chi+n_{e}^{2} \cos ^{2} \chi} .
$$

The relationship (3) generalized for an oblique incidence angle $\alpha \neq 0$ [5] related to the ring structure becomes

$$
\tan \phi^{\prime}=\frac{\left(n_{e}^{2}-n_{o}^{2}\right) \tan \left(\chi^{\prime}-\alpha\right)}{n_{e}^{2}+n_{o}^{2} \tan ^{2}\left(\chi^{\prime}-\alpha\right)}=\frac{\left(n_{e}^{2}-n_{o}^{2}\right) \tan \left(\chi^{\prime \prime}\right)}{n_{e}^{2}+n_{o}^{2} \tan ^{2}\left(\chi^{\prime \prime}\right)}
$$

In this case, the refractive index $n$ and the split angle $\phi^{\prime} \neq$ $\phi$ will change with the incidence angle $\alpha$; furthermore, we observe that $n_{e}$ and $n_{o}$ are the same as in the normal incidence case (incidence along the $z$-direction) of the thin-film multilayer structure.

The geometrical analogy will be obtained by fixing the $n_{e}$ and $n_{o}$ values [and consecutively, the $n_{e^{-}}$and $n_{o}$-axis of Fig. 3(a)] and by choosing a specific incidence angle $\alpha$ of the wave vector applied to the ring structure. The refractive index ellipse, obtained from the wavefront propagation, is characterized by the $n_{e}$ and $n_{o}$ fixed values, and by the index $n^{\prime}=n_{e}\left(\chi^{\prime}\right)=\mathbf{O B}^{\prime}$, which changes with the incidence angle $\alpha$ or, equivalently, with the $\chi^{\prime}$ orientation of the thin-film multilayer structure. Fig. 3(b) shows the generic refractive index ellipse at a particular propagation time for the concentric ring structure where the fictitious axis defines all the refractive indexes $n_{e}\left(\chi^{\prime}\right)$ obtained by varying the incidence angles $\alpha$ [5]. The equivalence between the thin-film and ring structures is obtained when the ellipses of Fig. 3(a) and (b) are equal. This is possible when the refractive indexes of the thin-film multilayer structure

$$
\begin{aligned}
& n_{e}^{2}=\frac{\left(h_{1}+h_{2}\right) n_{1}^{2} n_{2}^{2}}{h_{1} n_{2}^{2}+h_{2} n_{1}^{2}} \\
& n_{o}^{2}=\frac{h_{1} n_{1}^{2}+h_{2} n_{2}^{2}}{h_{1}+h_{2}}
\end{aligned}
$$

are equal to the following refractive indexes related to the DR structure:

$$
\begin{aligned}
n_{e}^{2} & =\frac{n_{1}^{2} n_{2}^{2}}{f_{1} n_{2}^{2}+f_{2} \varepsilon_{1}} \\
n_{o}^{2} & =f_{1} n_{1}^{2}+f_{2} n_{2}^{2}
\end{aligned}
$$




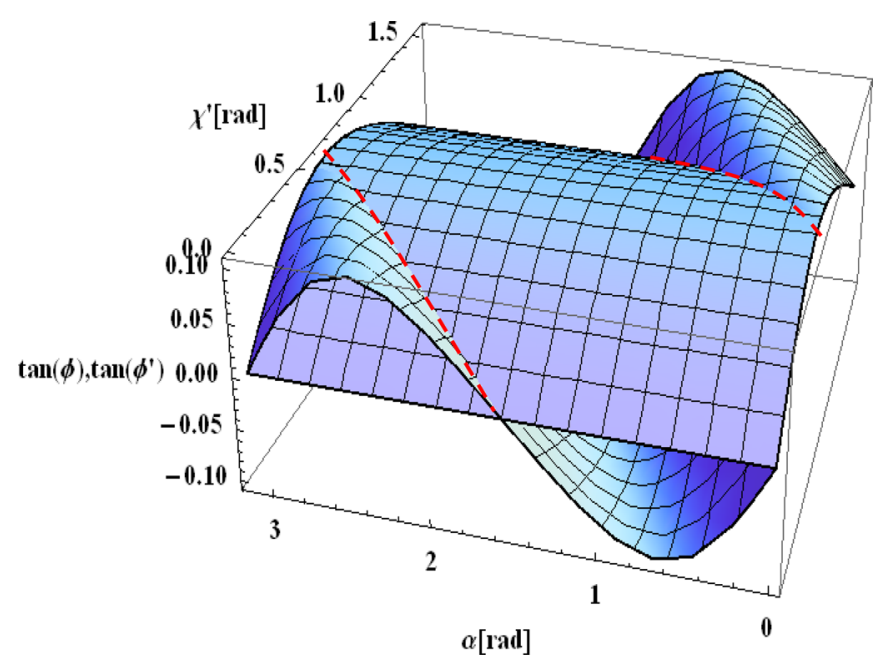

Fig. 5. Solution $\alpha\left(\chi^{\prime}\right)$ that satisfies the condition $\phi^{\prime}=\phi$ for $h_{1}=$ $0.25 \mu \mathrm{m}$ and $h_{2}=0.25 \mu \mathrm{m}$. The solutions are obtained by the intersection between the functions $\tan (\phi)$ and $\tan \left(\phi^{\prime}\right)$.

for a particular value of incidence angle $\alpha$ and positions $\mathbf{P}$ [see Fig. 2(b)], with $f_{1}=1-p$ and $f_{2}=p$ representing the dielectric filling factors [2] (fractions of the total space occupied by two dielectric materials) calculated for a particular incidence angle $\alpha$ and position of the point $\mathbf{P}$ which defines the segment $\mathbf{O P}$ in Fig. 2 (b). The position $\mathbf{P}$ and the angle $\alpha$ will define the exact position of the source $\mathrm{S}$. Moreover, the incidence angle $\alpha$ must simultaneously verify the condition $\phi^{\prime}=\phi$ in order to provide the same value $n^{\prime}=\mathbf{O B}^{\prime}=n=\mathbf{O B}=n_{e}\left(\chi^{\prime}\right)$. These conditions allow to obtain the same refractive index ellipse at the same propagation time $\Sigma^{\prime}$. In our theoretical model, the filling factors $f_{1}$ and $f_{2}$ of the ring structure are calculated by

$$
\begin{gathered}
f_{1}=\frac{\sum_{n} d_{n 1}}{\sum_{n} d_{n 1}+\sum_{m} d_{m 2}} \\
f_{2}=\frac{\sum_{n} d_{n 2}}{\sum_{n} d_{n 1}+\sum_{m} d_{m 2}}
\end{gathered}
$$

where $d_{n 1}$ and $d_{m 2}$ [see Fig. 1(b)] are the segments defined by the propagation direction of the $\mathbf{K}$ vector evaluated through the intersection between the line corresponding to the wave vector (line of equation $x=(z+\mathrm{OP}) \tan (\alpha))$ and the circles that define the rings. Equation (8), when applied to the multilayer of Fig. 1(a), becomes

$$
\begin{aligned}
& f_{1}=\frac{h_{1}}{h_{1}+h_{2}} \\
& f_{2}=\frac{h_{2}}{h_{1}+h_{2}} .
\end{aligned}
$$

Moreover, we observe that by considering more DRs, or, different central cavity sizes, the point $\mathrm{P}$ [see the intersection of the ordinary ray direction and the $z$-axis position of Fig. 2(b)] and the incidence angle $\alpha$ will change in order to obtain the same $n_{e}$ and $n_{o}$ values of the corresponding thin-film multilayer structure. The point $\mathrm{P}$ and the angle $\alpha$ will univocally define the propagation direction of the source.

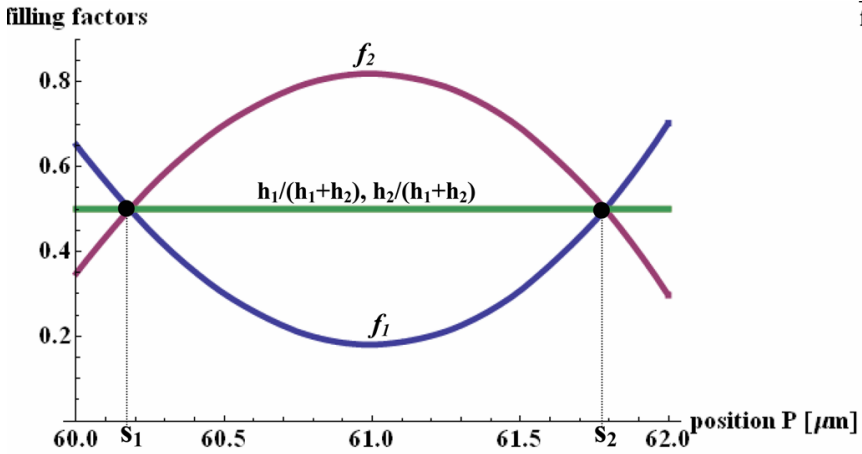

Fig. 6. Solutions $\mathrm{s}_{1}, \mathrm{~s}_{2}$ of the $\mathrm{P}$ positions for an incidence angle $\alpha=\pi / 3$ $\mathrm{rad}$ (corresponding to a value $\chi^{\prime}$ of $1.25 \mathrm{rad}$ ). The radius of the central cavity is $A=60 \mu \mathrm{m}$, and the ring thicknesses are $h_{1}=0.25 \mu \mathrm{m}$ and $h_{2}=0.25 \mu \mathrm{m}$.

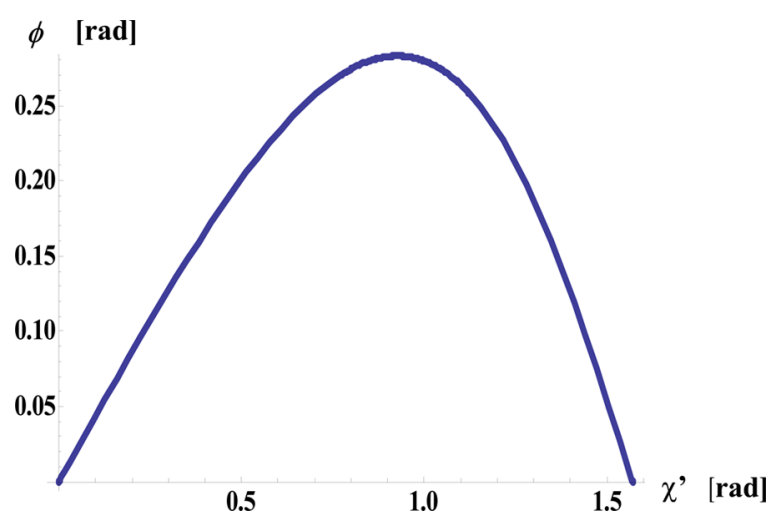

Fig. 7. Split angle $\phi$ versus $\chi^{\prime}$ orientation of the ring structure of Fig. 1(b), with $h_{1}=0.25 \mu \mathrm{m}$ and $h_{2}=0.25 \mu \mathrm{m}$.

\section{Examples of ANALogy Between Thin-Film Parallel DieleCtric Plates AND DiEleCtRIC CONCENTRIC RINGS}

In the case of thin-film parallel dielectric plates, two types of thin dielectric films with low $\left(n_{1}\right)$ and high $\left(n_{2}\right)$ refractive indexes alternate periodically. The high dielectric contrast provides a high difference between the ordinary and the extraordinary refractive index values: this allows to better distinguish the degenerate modes generated by the artificial anisotropy [2]. The thicknesses of the layers are sufficiently small as compared to the optical wavelength $\lambda_{0}$, and, consecutively, the period is in the order of $\lambda_{0}$. The optical of the artificial anisotropic dielectric is orthogonal to the laminated layers and forms an angle $\chi^{\prime}$ with the $z$-propagating direction [see Fig. 2(a)]. The layers of thicknesses $h_{1}$ and $h_{2}$ generate, in the multilayered structure, two polarized rays, associated to the ordinary (parallel to the layers) and the extraordinary (orthogonal to the layers) electric field components $\mathbf{E}_{o}$ and $\mathbf{E}_{e}$, respectively. The induced artificial anisotropy splits these two components of an angle $\phi$ [5]. Concerning the ring configuration of Fig. 1(a), we obtain the same optical behavior of the thin-film multilayer structure by constructing an equivalent circular structure with central cavity. The equivalence is proved by assuming particular physical conditions: the same birefringence effect of Fig. 2(a) is obtained for the equivalent circular device by means of particular incidence angle $\alpha$ and position of the source $\mathrm{S}$ of Fig. 2(b). The goal 


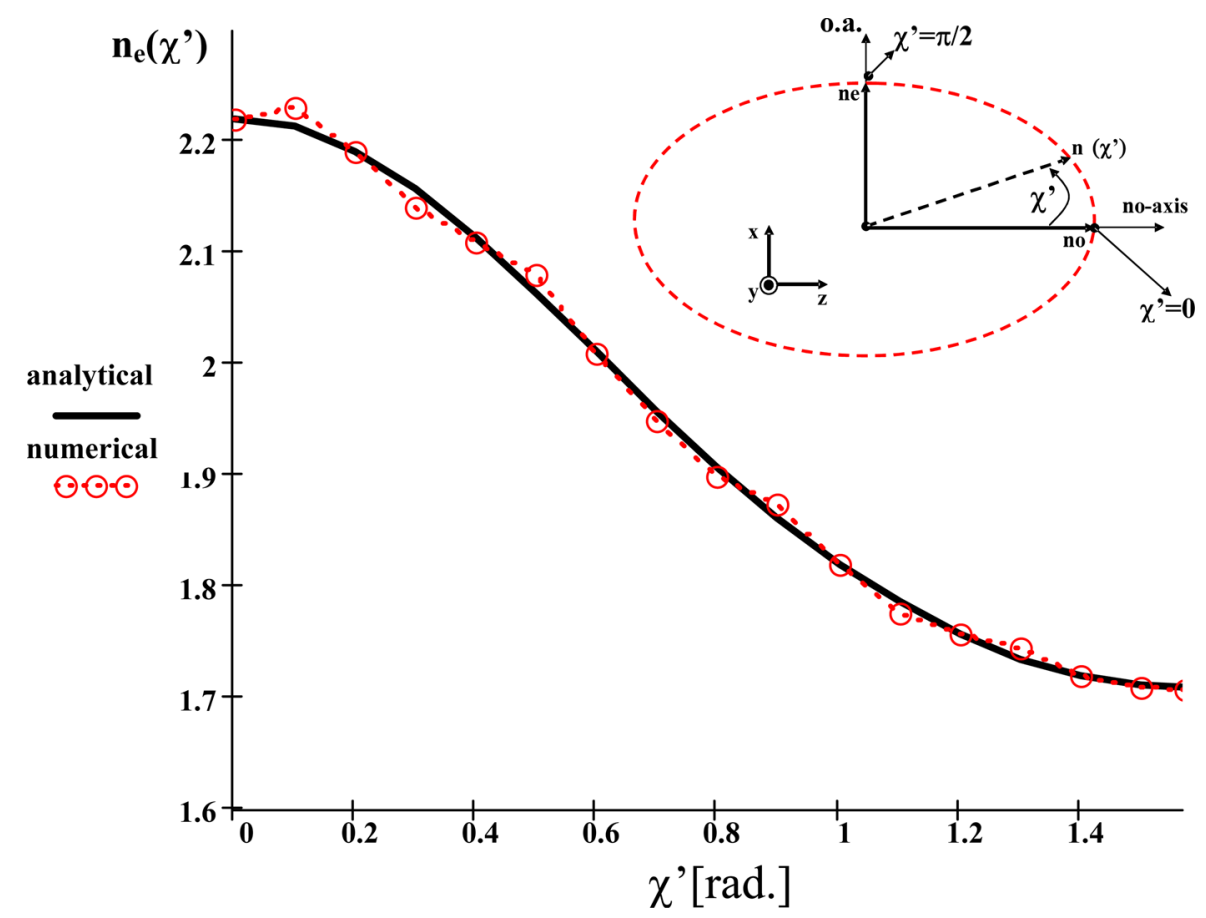

Fig. 8. Extraordinary refractive index $n_{e}\left(\chi^{\prime}\right)$ versus the optical axis orientation $\chi^{\prime}$. The analytical plot refers to the multilayer structure of Fig. 1(a) with $h_{1}=0.2 \mu \mathrm{m}, h_{2}=0.1 \mu \mathrm{m}$, and $A=40 \mu \mathrm{m}$. The numerical (FORTRAN code implementation) results are obtained for the equivalent ring structure through the possible combinations of incidence angles $\alpha$ and positions P. Inset: extraordinary refractive index ellipse.

(a)

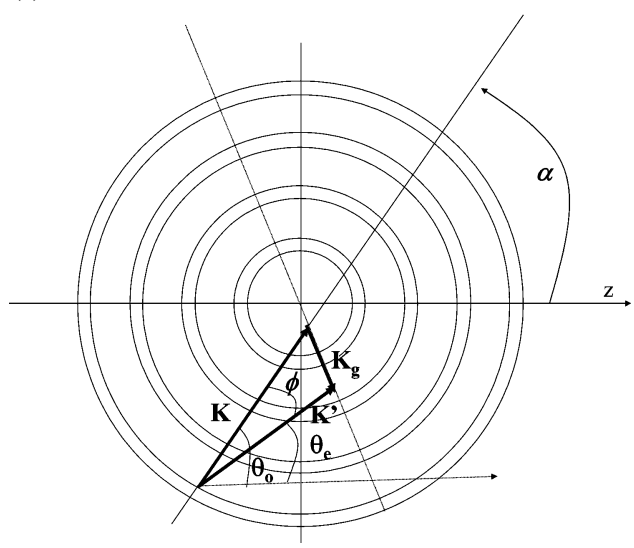

(b)

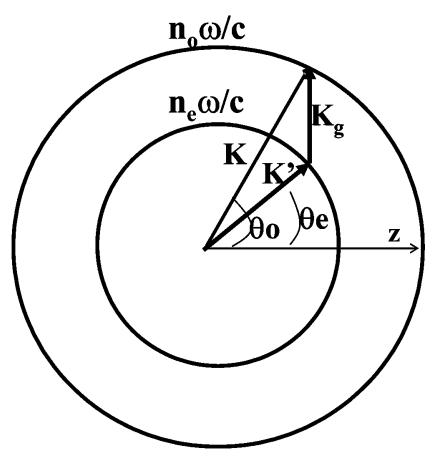

Fig. 9. (a) Anisotropic Bragg scattering in DRs. (b) Momentum wave vectors.

of this section is to find these incidence angles and positions through the analysis of the wavefront propagation and through the geometrical construction of the refractive index ellipse that provides the ordinary and the extraordinary refractive indexes associated to the ordinary field and the extraordinary field, respectively. Assuming a well-defined orientation of the source, the equivalent ring structure will be characterized by the same refractive indexes, thicknesses, split angle $\phi$, and optical axis angle $\chi^{\prime}$ of a thin-film parallel equivalent structure. The wave vector $\mathbf{K}$, with incidence angle $\alpha$, generates, in the circular crystal, the ordinary electric field component $\mathbf{E}_{o}$ (characterized by $n_{o}$ refractive index) and the extraordinary one $\mathbf{E}_{e}$ (characterized by $n_{e}$ refractive index) that are tangential and radial to the rings, respectively. The analog behavior of the thin-film multilayer structure is performed by the geometrical rotation of the equivalent dielectric multilayer structure around the symmetry $y$-axis of the circular structure [6]. As an application of the theoretical modeling in the 2-D space, we consider the $\mathrm{Si}$ $\left(n_{1}=3.24\right) / \mathrm{SiO}_{2}\left(n_{2}=1.465\right)$ layered structure reported in Fig. 2(a). The equivalent $\mathrm{Si} / \mathrm{SiO}_{2}$ DR structure is characterized by a $\mathrm{Si}$ central cavity with radius $A$, and ring thicknesses $h_{1}$ and $h_{2}$, as shown in Fig. 2(b). For a generic $\chi^{\prime}$ angle of the optical axis related to the thin parallel plates, it is possible to evaluate the set of solutions that satisfy (6) and (7). The same $n_{e}$ and $n_{o}$ values of the thin-film multilayer structure will be obtained by the evaluation of the filling factors $f_{1}$ and $f_{2}$ for different combinations of OP positions and $\alpha$ angles of the $\mathbf{K}$ vector of Fig. 2(b). Fig. 4 shows the geometrical locus 


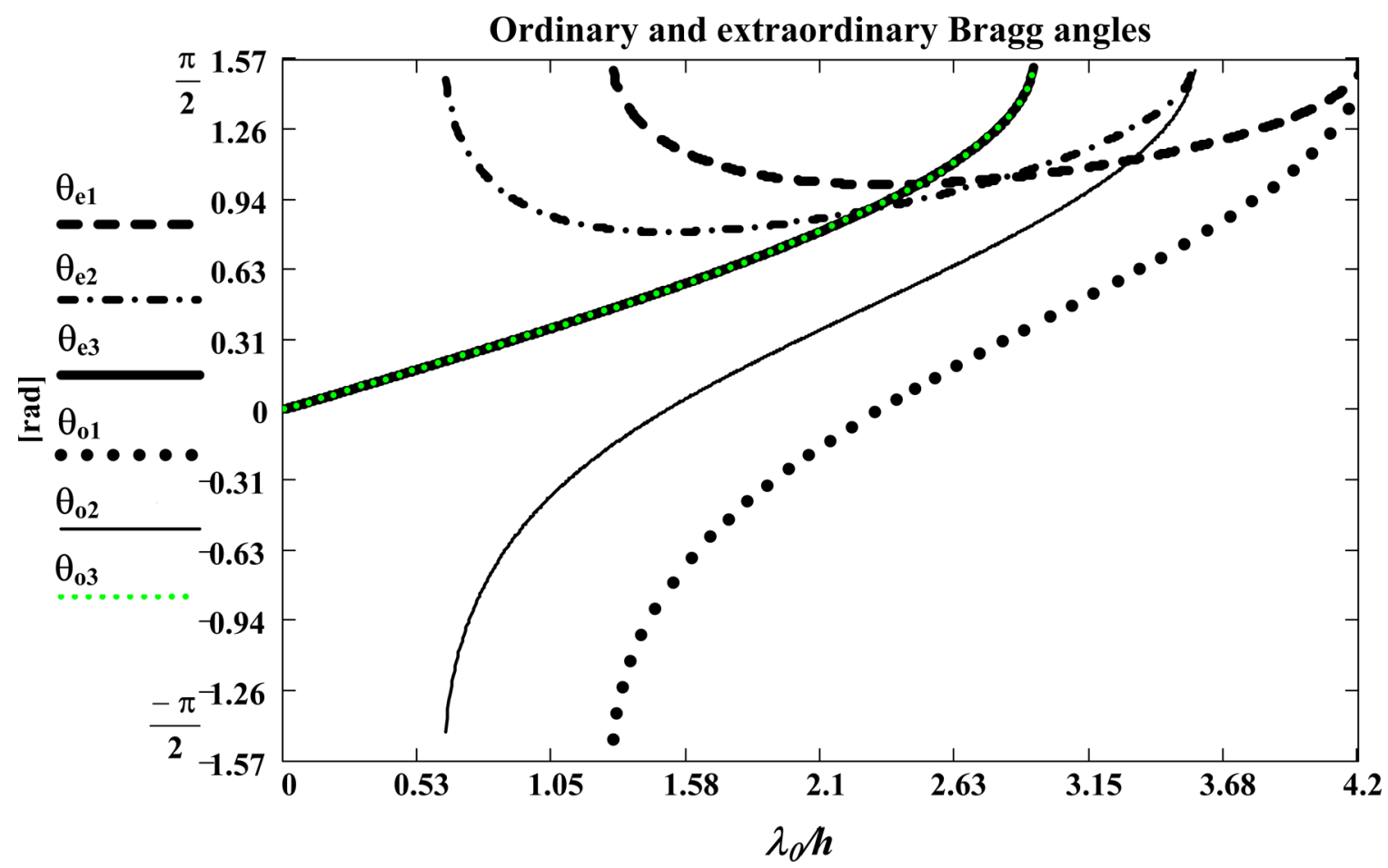

Fig. 10. Ordinary $\theta_{o}$ and extraordinary $\theta_{e}$ angles as functions of the ratio between the working wavelength $\lambda_{0}$ and the radial period $h$ in the case of concentric $\mathrm{SiO}_{2}$ rings $\left(n_{1}=1.465\right)$ and Si rings $\left(n_{2}=3.24\right)$ for $\alpha=\pi / 3 \mathrm{rad}, \chi^{\prime}=1.25 \mathrm{rad}, \mathrm{OP}=60.18 \mu \mathrm{m}\left(\theta_{e} 1\right.$ and $\theta_{o 1}$ plots $)$ for $\alpha=\pi / 6, \chi^{\prime}=0.93 \mathrm{rad}$, $\mathrm{OP}=58 \mu \mathrm{m}\left(\theta_{e 2}\right.$ and $\theta_{o 2}$ plots $)$ and for $\chi^{\prime}=0, \mathrm{OP}=0$ ( $\theta_{e 3}$ and $\theta_{o 3}$ plots). The ring structure is characterized by $h_{1}=0.25 \mu \mathrm{m}, h_{2}=0.25 \mu \mathrm{m}$, and $A=60 \mu \mathrm{m}$.

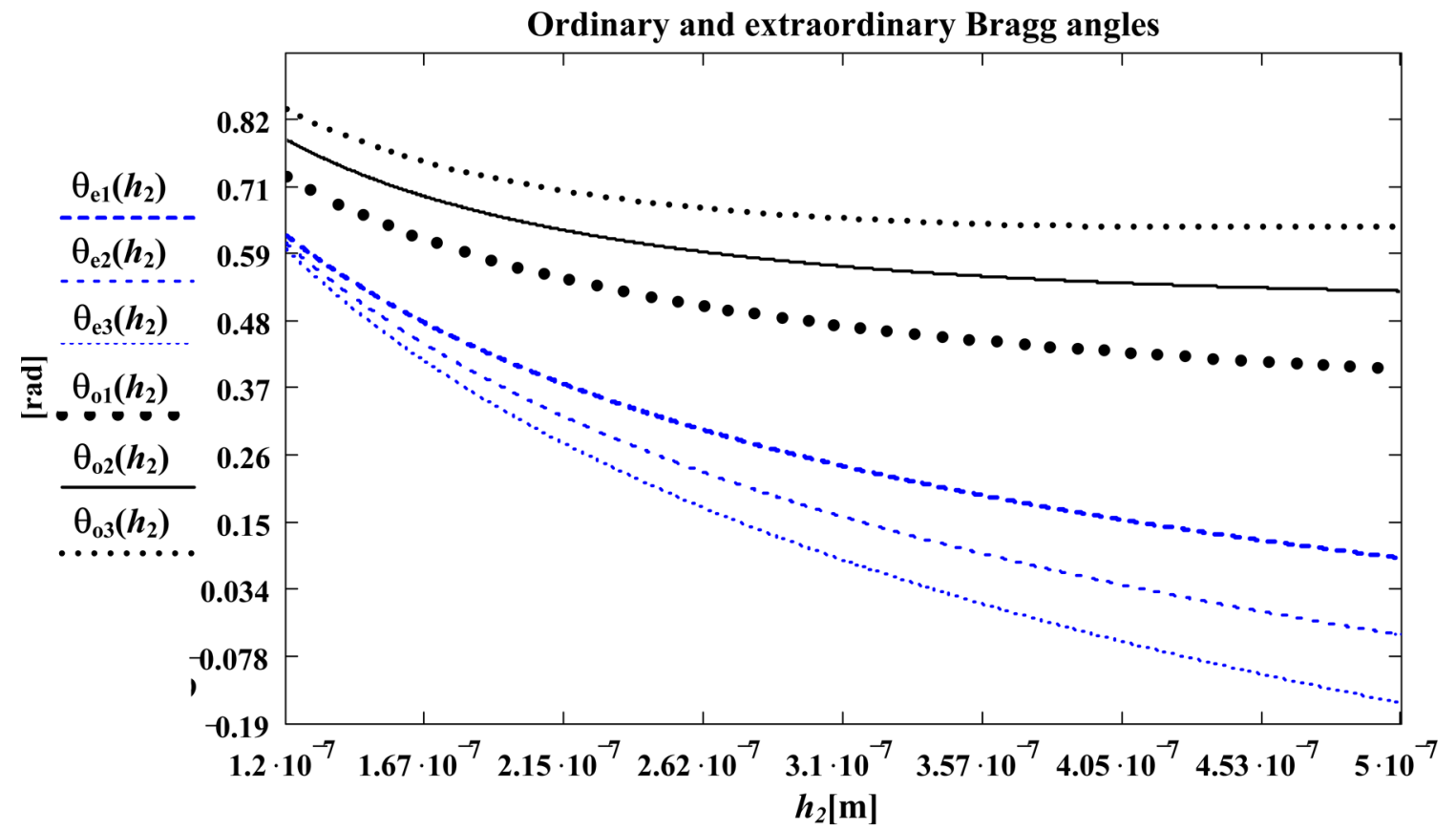

Fig. 11. Ordinary $\theta_{o}$ and extraordinary $\theta_{e}$ angles as function of $h_{2}$ at the working wavelength $\lambda_{0}=0.98 \mu \mathrm{m} \mathrm{in} \mathrm{the} \mathrm{case} \mathrm{of} \mathrm{concentric} \mathrm{SiO} \mathrm{O}_{2}$ rings $\left(n_{1}=1.465\right)$ and Si rings $\left(n_{2}=3.24\right)$ for $\chi^{\prime}=\pi / 6 \mathrm{rad}\left(\theta_{e 1}\right.$ and $\theta_{o 1}$ plots $)$, for $\chi^{\prime}=\pi / 4 \mathrm{rad}\left(\theta_{e 2}\right.$ and $\theta_{o 2}$ plots $)$ and for $\chi^{\prime}=\pi / 3 \mathrm{rad}\left(\theta_{e 3}\right.$ and $\theta_{o 3}$ plots $)$. The ring structure is characterized by $h_{1}=0.25 \mu \mathrm{m}$ and $A=60 \mu \mathrm{m}$. 
solution of (8) and (9) regarding $f_{1}$ and $f_{2}$ values versus the incidence angle $\alpha$ and position point P of Fig. 2(b). The plots reported in Fig. 4 are related to different ring structures: one with $h_{1}=0.01 \mu \mathrm{m}, h_{2}=0.01 \mu \mathrm{m}$, and $A=1 \mu \mathrm{m}$, and the other one with $h_{1}=0.25 \mu \mathrm{m}, h_{2}=0.25 \mu \mathrm{m}$, and $A=60 \mu \mathrm{m}$. The intersections (see dashed line of Fig. 4) between the calculated filling factors of the ring structure and the thin-film multilayer structure provide the locus solutions $(\alpha, \mathrm{OP})$ of the equivalence. Both the analyzed structures show that the point $\mathrm{P}$ is correlated to cavity radius $A$ : the $\mathrm{P}$ position solution is characterized by a value of the same order of the central cavity radius $A$. By applying the condition $\phi^{\prime}=\phi$ to (3) and (5), we find the relationship $\alpha\left(\chi^{\prime}\right)$, which provides the corresponding $\chi^{\prime}$ value of the equivalent multilayer structure (see dashed lines of Fig. 5). This $\alpha\left(\chi^{\prime}\right)$ relationship, reported in Fig. 5, combined with Fig. 4, provides the set of solutions $\alpha$, OP, and $\chi^{\prime}$ of the equivalent structures of Fig. 1(a) and (b). By considering, for example, an incidence angle $\alpha=\pi / 3$ rad related to a ring structure with $h_{1}=0.25 \mu \mathrm{m}$, $h_{2}=0.1 \mu \mathrm{m}$, and $A=60 \mu \mathrm{m}$, we find through Fig. 5 the corresponding value of the multilayer structure of $\chi^{\prime}=1.25 \mathrm{rad}$ and through Fig. 6 the corresponding position $\mathrm{P}$ solution of the equivalence (in this case, $\mathrm{OP}=60.18 \mu \mathrm{m}$ and $\mathrm{OP}=61.78 \mu \mathrm{m}$ ). By considering these conditions, the relationship between the split angle $\phi$ and the optical axis orientation $\chi^{\prime}$ is given in Fig. 7. The equivalence is proved by comparing the numerical results of the DR (FORTRAN code implementation) with the analytical ones related to the equivalent thin-film parallel plates: Fig. 8 shows the good agreement between analytical and numerical results related to the extraordinary refractive index evaluation. The numerical results of Fig. 8 are obtained through the possible combinations of incidence angles $\alpha$ and positions $\mathrm{P}$.

\section{DIELECTRIC RINGS AS A BRAGG REFLECTOR: ORDINARY AND EXTRAORDINARY BRAGG ANGLES}

The concentric rings generate, as in the dielectric multilayer structure, an ordinary and an extraordinary optical ray. For particular incidence angle $\alpha$ in the $z-x$ plane, the ordinary and the extraordinary rays are characterized by momentum conservation that defines the Bragg diffraction condition. This condition provides the maximum intensity of these two generated modes (ordinary and extraordinary modes) as a result of constructive interference. The ordinary $\theta_{o}$ and the extraordinary $\theta_{e}$ Bragg angles are obtained by the following considerations. The circular structure presents different gratings with the angle $\alpha$ varying in the plane as reported in Fig. 9(a), so that the light diffraction by gratings can be pictured as an interaction process between the incident wave and the diffracted wave [8]-[10]. In a birefringent medium, the refractive index associated with a light beam is dependent on the propagation direction. The diffracted light beam propagates in a direction different from the incident beam. For this purpose, we can associate the ordinary index $n_{o}$ with the incident beam (wave vector $|\mathbf{K}|=n_{o} \omega / c$ ) and the extraordinary index $n_{e}$ with the diffracted beam (wave vector $\left.\left|\mathbf{K}^{\prime}\right|=n_{e}\left(\chi^{\prime}\right) \omega / c\right)$. Momentum conservation requires that the incident wave vector, the diffracted wave vector, and the grating wave vector of the ring structure $\mathbf{K}_{g}(2 \pi / h)$ form the triangle shown in Fig. 9(b), where $h$ indicates the sum $h_{1}+h_{2}$. Let $\theta_{o}$ and $\theta_{e}$ be the angles between the light beams and the $\mathbf{K}_{g}$ wave vector of the grating. The Bragg diffraction conditions are obtained from the triangle of Fig. 9(b) as [5]

$$
\begin{aligned}
2 K \sin \theta_{o} & =K_{g}-\frac{K^{\prime^{2}}-K^{2}}{K_{g}} \\
2 K^{\prime} \sin \theta_{e} & =K_{g}+\frac{K^{\prime^{2}}-K^{2}}{K_{g}}
\end{aligned}
$$

or equivalently, for a general $\chi^{\prime}$ orientation in the plane $x-z$

$$
\begin{aligned}
2 h \sin \theta_{o} & =\frac{\lambda_{0}}{n_{o}}-\frac{h^{2}}{n_{o} \lambda_{0}}\left(n_{e}^{2}\left(\chi^{\prime}\right)-n_{o}^{2}\right) \\
2 h \sin \theta_{e} & =\frac{\lambda_{0}}{n_{e}(\chi)}-\frac{h^{2}}{n_{e}(\chi) \lambda_{0}}\left(n_{e}^{2}\left(\chi^{\prime}\right)-n_{o}^{2}\right)
\end{aligned}
$$

according to (12) and (13), the angles of both light beams are functions of $\lambda_{0} / h$ when $n_{o}$ and $n_{e}\left(\chi^{\prime}\right)$ are fixed. Bragg diffraction is possible only when [5]

$$
\left|n_{o}-n_{e}\left(\chi^{\prime}\right)\right| \leq \frac{\lambda_{o}}{h} \leq\left|n_{o}+n_{e}\left(\chi^{\prime}\right)\right|
$$

and the angles $\theta_{o}$ and $\theta_{e}$ are real. We observe in the case $\mathrm{P}=\mathrm{O}$ that the extraordinary refractive ellipse degenerate in a circumference with radius $n_{o}$ and $\theta_{o}=\theta_{e}$ ( $\mathbf{K}^{\prime}$ and $\mathbf{K}$ are collinear) according to conservation of momentum reported in Fig. 9(b). In Fig. 10, we show the Bragg angles obtained for different wavelengths $\lambda_{0}$ regarding the $\mathrm{Si} / \mathrm{SiO}_{2}$ ring structure of Fig. 2(b) for different angles $\chi^{\prime}$. Moreover, Figs. 11 and 12 report the Bragg angles $\theta_{o}$ and $\theta_{e}$ for different angles $\chi^{\prime}$ of the equivalent multilayer structure, by varying the thickness $h_{2}$ and by fixing the working optical wavelengths to $\lambda_{0}=0.98 \mu \mathrm{m}, \lambda_{0}=1.31 \mu \mathrm{m}$, and $\lambda_{0}=1.55 \mu \mathrm{m}$, respectively. We observe that the corresponding $\alpha$ and $\mathrm{OP}$ values of the equivalent ring structure are evaluated through the plots of Figs. 4 and 5 obtained for each couple of $h_{1}$ and $h_{2}$ values. We validate the Bragg theory through the time-domain modeling. In particular, we model the $\mathrm{Si} / \mathrm{SiO}_{2}$ DRs by a commercial 2-D FDTD software (RSOFT Design Group). The position and the orientation of the source are defined by the analytical model. The ordinary and extraordinary intensities at the output are displayed on a time monitor orthogonal to the ordinary ray. The split angle $\phi=\theta_{o}-\theta_{e}$ is evaluated through geometrical considerations that take into account the distance of the source and the distance between the maxima of the electric fields associated to the ordinary and the extraordinary rays, respectively. Finally, as validation of the previous comparison, we simulate the same structure by means of HPF modeling typically used to model dielectric interfaces of multilayered optical waveguides [16]. This tool, combined with the commercial FDTD software, verifies the analytical results related to the Bragg theory. In particular, Fig. 13 shows the calculated and the simulated split angles $\phi$ versus $h_{2}$ by considering $\lambda_{0}=0.98 \mu \mathrm{m}, h_{1}=0.25 \mu \mathrm{m}$, and $A=60 \mu \mathrm{m}$. An average error of 0.035 rad between the FDTD simulation and the calculation is observed. The average error is reduced to 0.005 rad by HPF simulation. Both the numerical simulations confirm the theoretical model results. Table I shows all parameters 

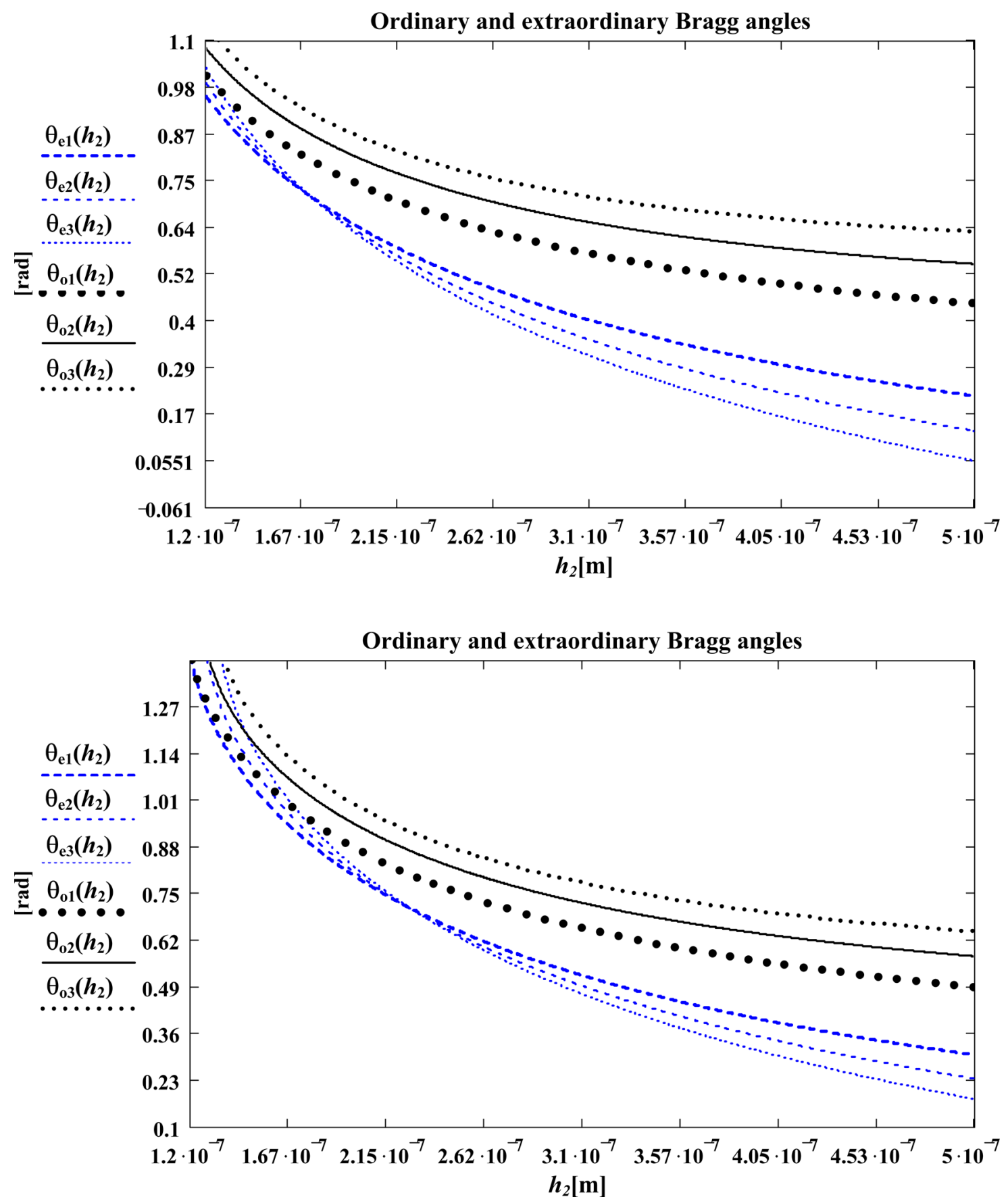

Fig. 12. (Above) Ordinary $\theta_{0}$ and extraordinary $\theta_{e}$ angles as function of $h_{2}$ at the working wavelength $\lambda_{0}=1.31 \mu \mathrm{m}$ in the case of concentric $\mathrm{SiO} \mathrm{O}_{2}$ rings $\left(n_{1}=1.465\right)$ and Si rings $\left(n_{2}=3.24\right)$ for $\chi^{\prime}=\pi / 6 \mathrm{rad}\left(\theta_{e 1}\right.$ and $\theta_{o 1}$ plots $)$, for $\chi^{\prime}=\pi / 4 \mathrm{rad}\left(\theta_{e 2}\right.$ and $\theta_{o 2}$ plots $)$ and for $\chi^{\prime}=\pi / 3 \mathrm{rad}\left(\theta_{e 3}\right.$ and $\theta_{o 3}$ plots). The ring structure is characterized by $h_{1}=0.25 \mu \mathrm{m}$ and $A=60 \mu \mathrm{m}$. (Below) Ordinary $\theta_{o}$ and extraordinary $\theta_{e}$ angles as function of $h_{2}$ at the working wavelength $\lambda_{0}=1.55 \mu \mathrm{m}$ in the case of concentric $\mathrm{SiO}_{2} \operatorname{rings}\left(n_{1}=1.465\right)$ and Si rings $\left(n_{2}=3.24\right)$ for $\chi^{\prime}=\pi / 6 \mathrm{rad}\left(\theta_{e 1}\right.$ and $\theta_{o 1}$ plots $)$, for $\chi^{\prime}=\pi / 4$ $\operatorname{rad}\left(\theta_{e 2}\right.$ and $\theta_{o 2}$ plots) and for $\chi^{\prime}=\pi / 3 \operatorname{rad}\left(\theta_{e 3}\right.$ and $\theta_{o 3}$ plots). The ring structure is characterized by $h_{1}=0.25 \mu \mathrm{m}$ and $A=60 \mu \mathrm{m}$.

obtained during the calculation of the split angles of Fig. 13 and the computational cost (CPU time) for a 1-GHz PC, $512 \mathrm{MB}$ RAM.

\section{Circular Photonic Crystal: Microcavity Resonator}

The GaAs CPhC is an unconventional kind of quasi-3-D photonic crystal with circular lattice pattern: it consists of air holes in a GaAs material ( $n=3.408)$ along circular concentric lines.
This particular circular geometry has peculiar behavior if compared with the traditional square and triangular lattices, but it is difficult to model by using conventional numerical approaches such as wave expansion method. If the cylindrical air holes are properly designed, the degenerate modes, generated in the circular structure, can be confined in a central microcavity region by increasing the power radiated above the microcavity. A proper tuning of the inner holes of Fig. 1(b) (holes shift and diameters 
TABLE I

CALCUlATED $\phi_{c}$ AND NUMERICAL SPlit ANGLES $\phi_{\text {FDTD AND }} \phi_{\text {HPF }}$

\begin{tabular}{|l|l|l|l|l|l|l|l|l|l|}
\hline$h_{2}[\mu \mathrm{m}]$ & $\begin{array}{l}\text { OP } \\
{[\mu \mathrm{m}]}\end{array}$ & $n_{o}$ & $n_{e}$ & $n_{e}\left(\chi^{\prime}\right)$ & $\phi_{c}$ [deg.] & $\begin{array}{l}\phi_{F D T D} \\
{[\mathrm{deg} .]}\end{array}$ & $\begin{array}{l}\phi_{H P F} \\
{[\mathrm{deg} .]}\end{array}$ & $\begin{array}{l}\text { CPU time } \\
\text { FDTD[sec.] }\end{array}$ & $\begin{array}{l}\text { CPU time } \\
\text { HPF[sec.] }\end{array}$ \\
\hline 0.1 & 59.6 & 2.128 & 1.666 & 1.700 & 0.220 & 0.181 & 0.220 & 4.4 & 1.9 \\
\hline 0.15 & 59.8 & 2.297 & 1.748 & 1.787 & 0.341 & 0.313 & 0.341 & 4.8 & 2.1 \\
\hline 0.2 & 60 & 2.420 & 1.822 & 1.863 & 0.455 & 0.421 & 0.455 & 5.2 & 2.4 \\
\hline 0.25 & 60.18 & 2.514 & 1.887 & 1.930 & 0.541 & 0.500 & 0.540 & 5.8 & 2.9 \\
\hline 0.3 & 60.4 & 2.588 & 1.947 & 1.991 & 0.622 & 0.591 & 0.621 & 6.2 & 3.1 \\
\hline 0.35 & 60.6 & 2.649 & 2.001 & 2.045 & 0.690 & 0.651 & 0.690 & 6.7 & 3.4 \\
\hline
\end{tabular}

The numerical values are obtained by 2-D-FDTD and 2-D HPF approaches. The ring structure is characterized by $h_{l}=0.25 \mu \mathrm{m}, A=60 \mu \mathrm{m}, \alpha=\pi / 3 \mathrm{rad}$,

$\chi^{\prime}=1.25 \mathrm{rad}$, and $\lambda_{0}=0.98 \mu \mathrm{m}$.

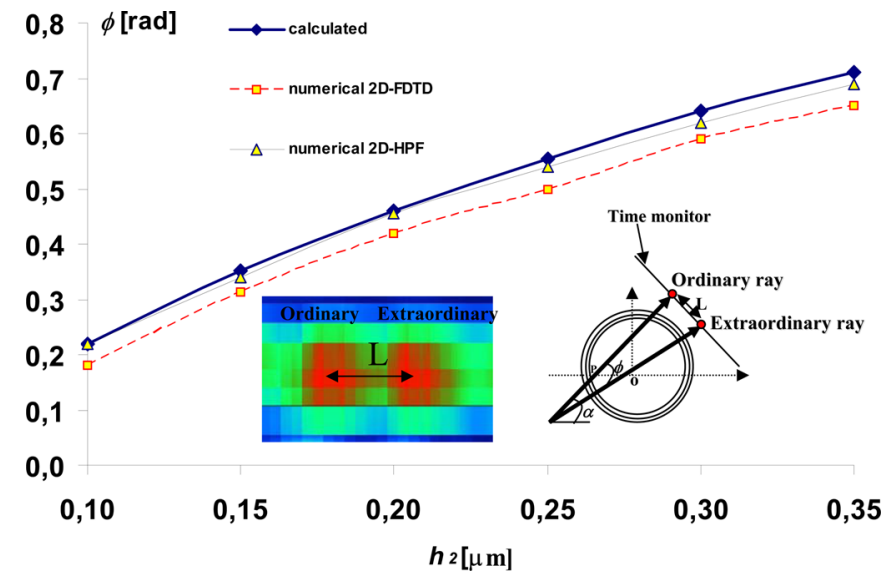

Fig. 13. Calculated and simulated split angles $\phi=\theta_{0}-\theta_{e}$ versus $h_{2}$ by considering Bragg condition. The ring structure is characterized by $h_{1}=0.25 \mu \mathrm{m}$, $A=60 \mu \mathrm{m}, \alpha=\pi / 3 \mathrm{rad}, \chi^{\prime}=1.25 \mathrm{rad}$, and $\lambda_{0}=0.98 \mu \mathrm{m}$. Inset: ordinary and extraordinary rays (intensity of the total electric field) displayed on the time monitor and numerical modeling.
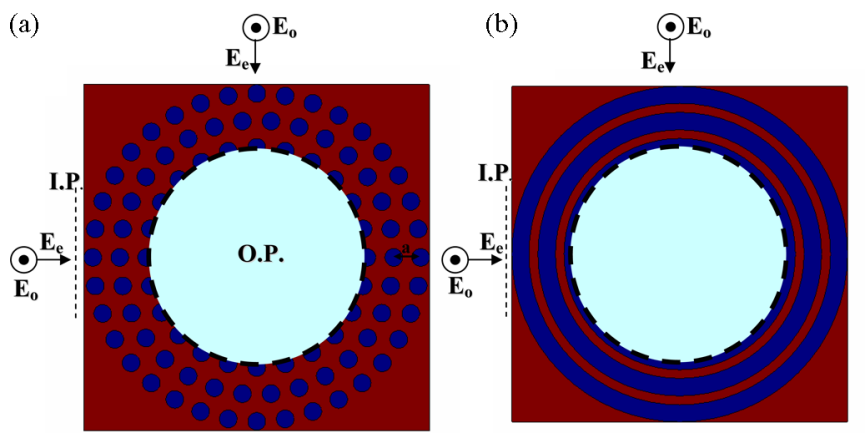

Fig. 14. FEM modeling. (a) CphC with air circular holes. (b) DRs approximation where the DRs thicknesses are equal to the outer holes diameter and the central DR has a thickness equal to the inner holes radius. The input port I.P. is placed on the excitation side and the output port O.P. is placed in the cavity region. The I.P. and the O.P. ports are indicated by dashed lines.

variation) can provide a high-intensity microcavity resonance. For this purpose, the inner holes have a reduced radius and are shifted along the radial outward direction, in order to improve the resonance properties. The analyzed $\mathrm{CPhC}$ is characterized by lattice constant $a=350 \mathrm{~nm}$ [see Fig. 14(a)], outer holes radius of $120 \mathrm{~nm}$, and GaAs slab thickness of $200 \mathrm{~nm}$.

We analyze the microcavity resonances of the degenerate mode by a 3-D FEM tool [17] typically used to design CPhCs. The modeling is based on the definition of the unit cell [17]

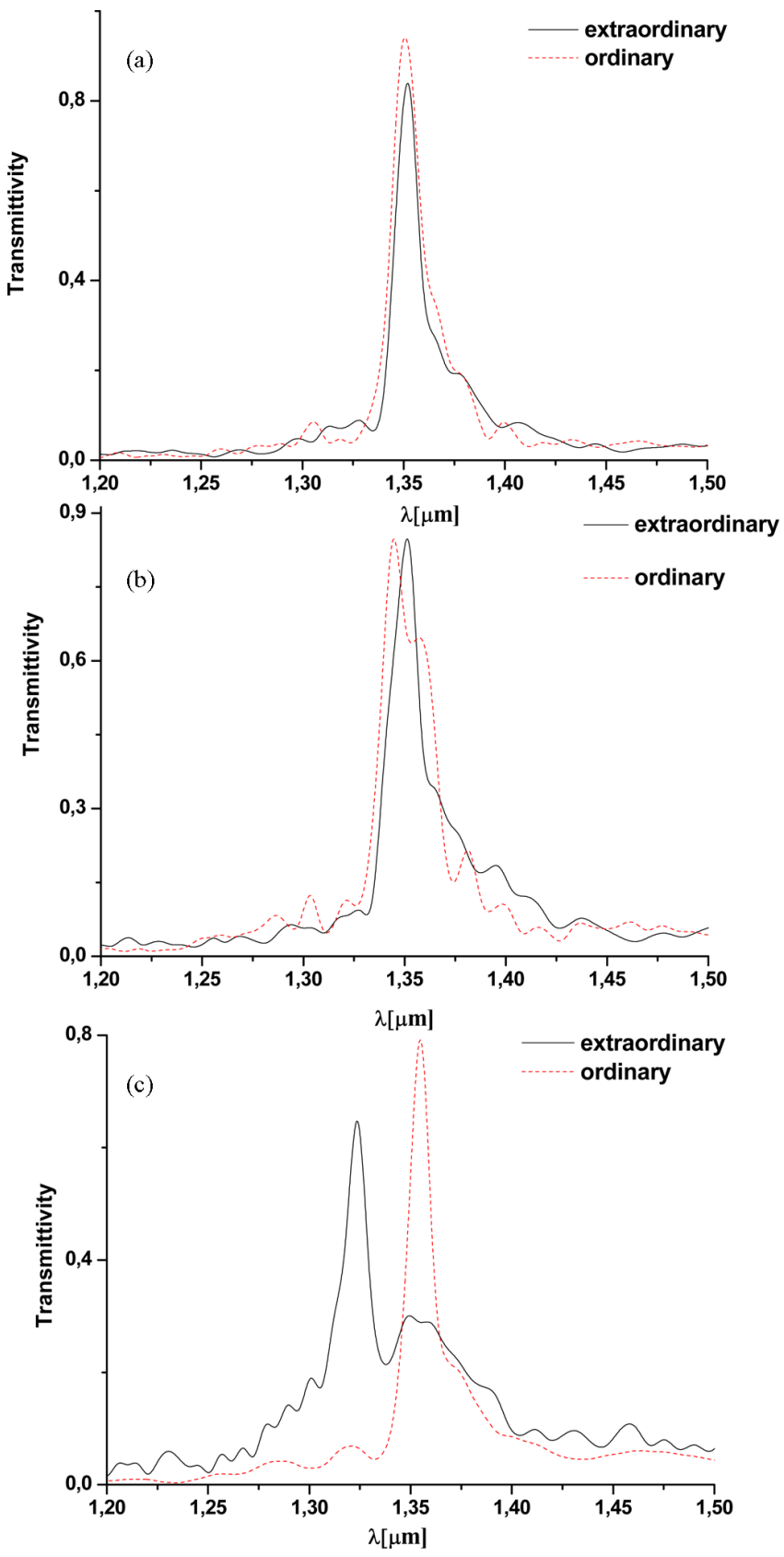

Fig. 15. FEM transmittivity of CphC. (a) Ordinary and extraordinary frequency response for inner holes radius of $80 \mathrm{~nm}$. (b) Ordinary and extraordinary frequency response for inner holes radius of $80 \mathrm{~nm}$. (c) Ordinary and extraordinary frequency response for inner holes radius of $70 \mathrm{~nm}$. The analyzed $\mathrm{CPhC}$ is characterized by lattice constant $a=350 \mathrm{~nm}$, outer holes radius $R=120 \mathrm{~nm}$, and GaAs slab thickness of $200 \mathrm{~nm}$. 
as a 3-D $\mathrm{CphC}(3 \mathrm{D}-\mathrm{CPhC})$. The unit cell is characterized by resonance field, radiation field, and scattering parameters. In the 3-D FEM modeling, the interface between all the 3-D objects and the background is a perfect "absorbing boundary" through which no energy may enter or exit. Wave ports are typically placed at this interface in order to provide a window that couples the model device to the external world. In our case, we set the ports as indicated in Fig. 14(a) and (b), where the input port is placed on the excitation side and the output port is placed above the central cavity region. In order to distinguish the ordinary mode and the extraordinary ones, we excite from the input port with different electric field polarizations: the $\mathbf{E}_{o}$ excitation will select the transmittivity (between the input and the output port) related to the ordinary mode and $\mathbf{E}_{e}$ excitation will characterize the transmittivity of extraordinary mode. In Fig. 15(a)-(c) are reported the ordinary and the extraordinary frequency responses by changing the inner hole radius of the $\mathrm{CPhC}$. We observe from Fig. 15 that the two modes are better distinguished for an inner holes radius of $70 \mathrm{~nm}$ : in this case, an extraordinary peak is observed at $\lambda=1.32 \mu \mathrm{m}$ and an ordinary peak is found around $\lambda=1.36 \mu \mathrm{m}$. The equivalent DR structure (also reported in Fig. 1(a), where the concentric air rings are the extended case of infinite air cylinders arranged in circular patterns) is formed with the DR thickness of the same dimension as the outer holes diameter and with inner DR thickness of the same dimension as the inner holes diameter. In Fig. 16 are reported the ordinary and the extraordinary transmittivities of the equivalent DR structure: also, in this case, the peaks associated to the ordinary and the extraordinary modes are well distinguished, but their positions are shifted with respect to the $\mathrm{CPhC}$ structure, as shown in Fig. 17(a) and (b). The peak shift highlights the limits of the ring approximation: in fact, the extraordinary refractive index of the $\mathrm{CPhC}$ oscillates around the value of the corresponding DR structure [6] by providing the shift of the frequency response. Moreover, Figs. 18 and 19 show the difference of the electric field confinement by comparing the $\mathrm{CPhC}$ and the equivalent DR structure: the function of the central microcavity is to better confine in a small region the ordinary and the extraordinary fields in order to increase the power radiated in the vertical $y$-direction. A high radiated power is obtained in membrane-type photonic $\mathrm{CPhC}$ : the air cladding thickness below the $\mathrm{CPhC}$ slab enhances the radiated power in the region above the microcavity [17]. We estimate this enhancement through the $Q$-factor. The $Q$-factor (FEM solution) is evaluated as [17]

$$
Q=2 \pi \operatorname{freq} \frac{U}{P}
$$

where $U$ is the energy stored inside the $\mathrm{CPhC}, P$ is the power lost through a domain that encloses the whole structure, and freq is the working frequency. The air cladding layer behaves as an air cavity resonator that, if properly sized (nominal depth as $\lambda_{0} / 2$ or odd multiples of $\lambda_{0} / 2$ ), should provide electromagnetic field enhancement of the GaAs waveguide layer. We observe from Fig. 20 that by varying the air cavity depth from the nominal value in steps of $20 \mathrm{~nm}$, the $Q$-factor will drastically decrease: this aspect provides the $Q$-factor sensitivity versus the air cavity

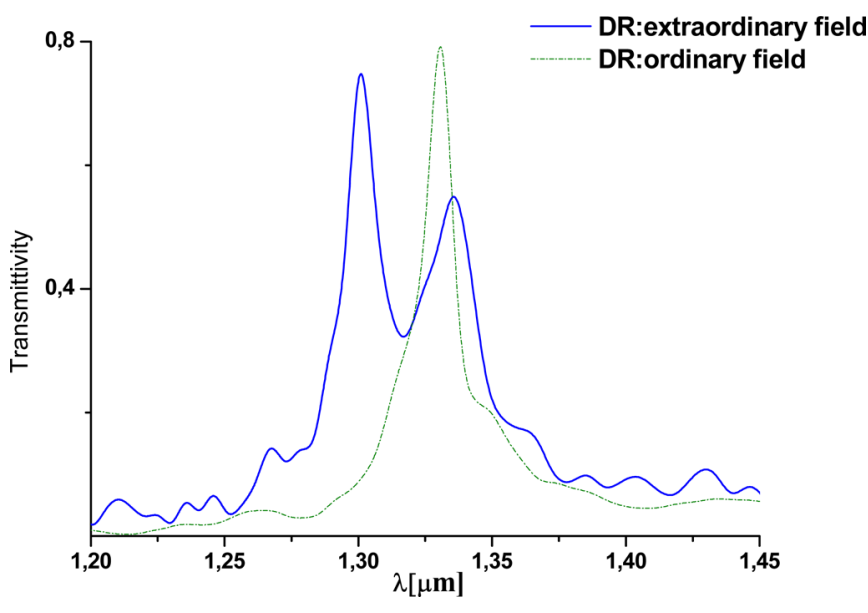

Fig. 16. FEM transmittivity DRs. Ordinary and extraordinary frequency response for DR thicknesses of $120 \mathrm{~nm}$. The inner DR is characterized by a thickness of $70 \mathrm{~nm}$.
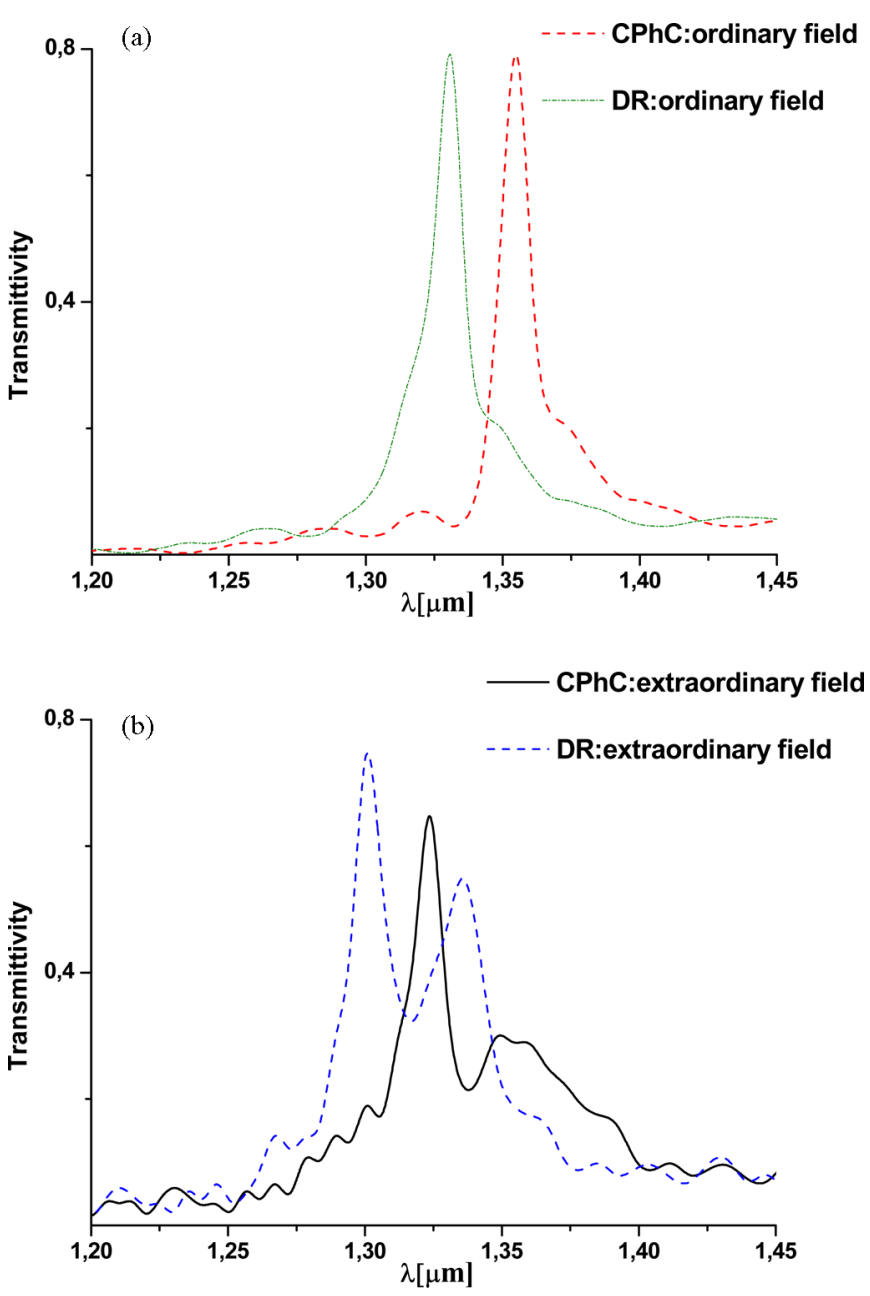

Fig. 17. Comparison of FEM transmittivities between the $\mathrm{CphC}$ and the corresponding DRs structure. (a) Ordinary frequency response for inner holes radius of $70 \mathrm{~nm}$ and for an inner DR with a thickness of $70 \mathrm{~nm}$. (b) Extraordinary frequency response for inner holes radius of $70 \mathrm{~nm}$ and for an inner DR with a thickness of $70 \mathrm{~nm}$. 


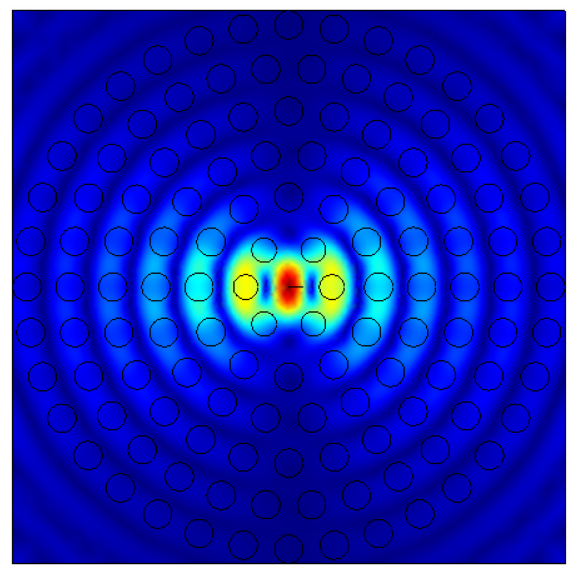

(a)

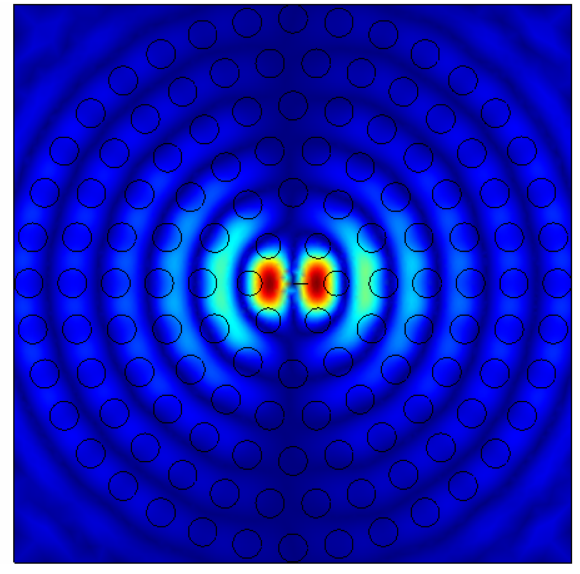

(b)

Fig. 18. FEM electric field displacement in CPhC. (a) Ordinary electric field confinement $\mathbf{E}_{o}$. (b) Extraordinary electric field confinement $\mathbf{E}_{e}$.

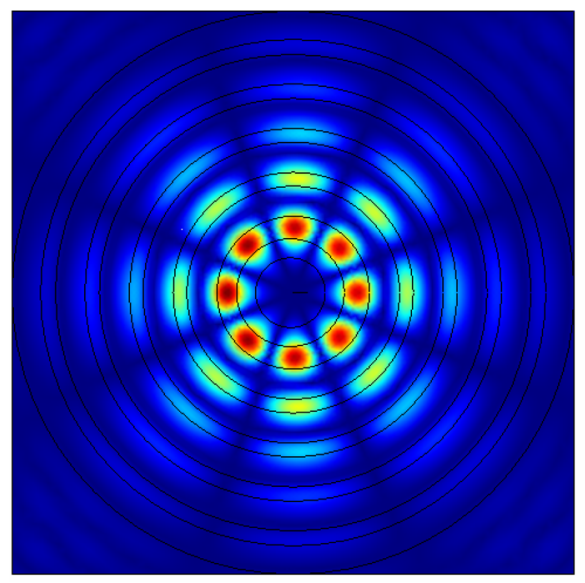

(a)

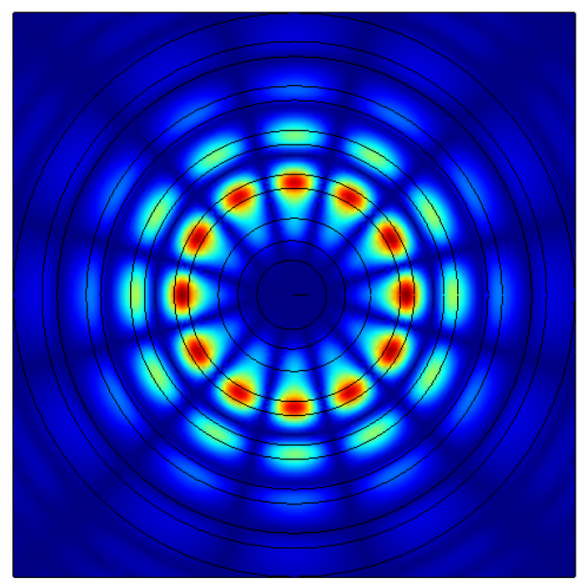

(b)

Fig. 19. FEM electric field displacement (WGM profiles) in DRs. (a) Ordinary electric field confinement $\mathbf{E}_{o}$. (b) Extraordinary electric field confinement $\mathbf{E}_{e}$.

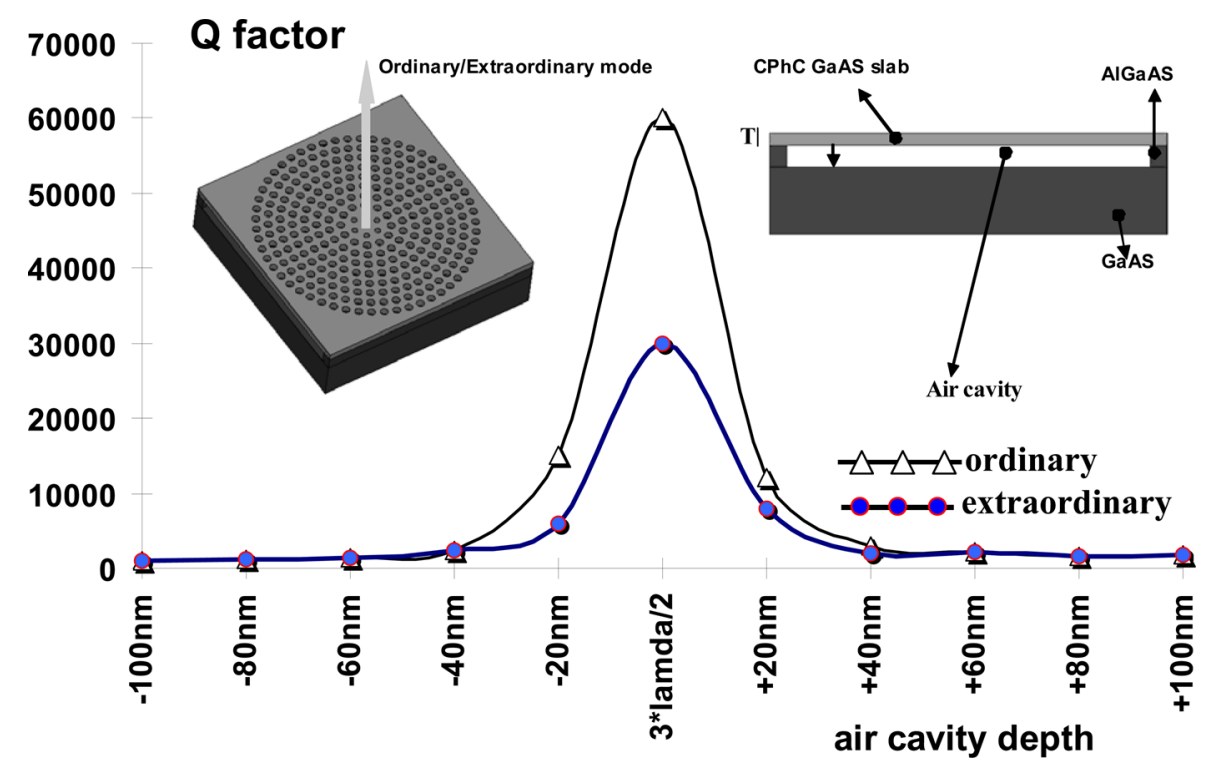

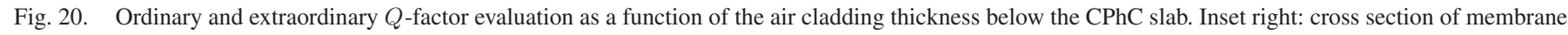
type $\mathrm{CPhC}$ with slab thickness $T=200 \mathrm{~nm}$. Inset left: schematic plot of the radiated power direction. 
thickness. We numerically found that for an air cavity depth of $3 \lambda_{0} / 2$, the $Q$-factor is of the order of $6 \times 10^{4}$ for the ordinary mode and of the order of $3 \times 10^{4}$ for the extraordinary mode.

\section{CONCLUSION}

We presented in this paper a theoretical model that provides the birefringent effects in $\mathrm{CphCs}$ at optical frequencies. The model, obtained through the geometrical synthesis of a thinfilm multilayer equivalent structure, explains the presence of degenerate modes inside the CphCs such as DRs photonic crystals and air holes CphCs. As applications of these degenerate modes, we present a $\mathrm{Si} / \mathrm{SiO}_{2}$ DR structure that behaves as a Bragg reflector and a GaAs membrane-type $\mathrm{CphC}$ that behaves as a microcavity resonator. The theory can be applied to a large number of applications such as polarization switching, filtering, and multiplexing.

\section{REFERENCES}

[1] F. Abelès, "Recherches sur la propagation des ondes electromagetique sinusoidales dans les milieux stratifes," Ann. Physiquevol., vol. 5, pp. 596640, 706-782, 1950.

[2] M. Born and E. Wolf, Eds., Principles of Optics, 6th ed. Cambridge, U.K.: Cambridge Univ. Press, 1980, ch. 14.

[3] B. Rossi, Optics. Reading, MA: Addison-Wesley, 1967, ch. 6.

[4] K. Shiraishi and K. Matsumura, "Fabrication of spatial walk-off polarizing film by oblique depositions," IEEE J. Quantum Electron., vol. 30, no. 10, pp. 2417-2420, Oct. 1994.

[5] A. Massaro, L. Pierantoni, and T. Rozzi, "Accurate analysis of wave propagation in negative uniaxial crystal," IEEE J. Quantum Electron., vol. 40, no. 6, pp. 821-829, Jun. 2004.

[6] A. Massaro, V. Errico, R. Cingolani, M. Devittorio, and A. Passaseo, "Negative uniaxial crystal behavior of circular photonic crystal," IEEE J. Quantum Electron., vol. 44, no. 12, pp. 1225-1231, Dec. 2008.

[7] A. D. Remenyuk, E. V. Astrova, T. S. Perova, V. A. Tolmachev, J. Vij, and A. Moore, "Large optical anisotropy in the structure of 1D photonic crystal fabricated by vertical etching of silicon," in Proc. ICTON 2003, Jun., vol. 1, pp. 269-272.

[8] P. Yeh, Introduction to Photorefractive Nonlinear Optics. New York: Wiley-Interscience, 1993, ch. 2.

[9] P. Yeh, Optical Waves in Layered Media. New York: Wiley, 1988.

[10] A. Yariv, Optical Waves in Crystals. New York: Wiley, 1984.

[11] P. T. Lee, T. W. Lu, and F. M. Tsai, "Octagonal quasi-photonic crystal single-defect microcavity with whispering gallery mode and condensed device size," IEEE Photon. Technol. Lett., vol. 19, no. 9, pp. 710-712, May 2007.

[12] P. T. Lee, T. W. Lu, J. H. Fan, and F. M. Tsai, "High quality factor microcavity lasers realized by circular photonic crystal with isotropic photonic band gap effect," Appl. Phys. Lett., vol. 90, pp. 151125-1151125-3, 2007.

[13] J. Scheuer and A. Yariv, "Coupled-waves approach to the design and analysis of Bragg and photonic crystal annular resonator," IEEE J. Quantum Electron., vol. 39, no. 12, pp. 1555-1562, Dec. 2003.

[14] J. Scheuer and A. Yariv, "Circular photonic crystal resonator," Phys. Rev. $E$, vol. 70, pp. 036603-1-036603-8, 2004.

[15] A. Tandaechanurat, S. Iwamoto, M. Nomura, N. Kumagai, and Y. Arakawa, "Increase of Q-factor in photonic crystal H1-defect nanocavities after closing of photonic bandgap with optimal slab thickness," Opt. Exp., vol. 16, no. 1, pp. 448-455, Jan. 2008.

[16] A. Massaro, M. Grande, R. Cingolani, A. Passaseo, and M. De Vittorio, "Design and modeling of tapered waveguide for photonic crystal slab coupling by using time-domain Hertzian potentials formulation," Opt. Exp., vol. 15, no. 25, pp. 16484-16499, Dec. 2007.

[17] A. Massaro, V. Errico, T. Stomeo, A. Salhi, R. Cingolani, A. Passaseo, and M. De Vittorio, "3D FEM modeling and fabrication of circular photonic crystal microcavity," J. Lightw. Technol., vol. 26, no. 16, pp. 2960-2968, Aug. 2008.

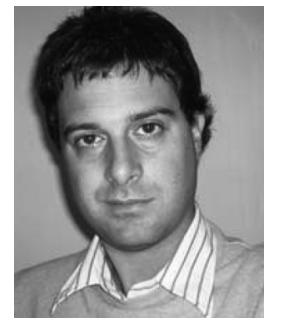

Alessandro Massaro received the Laurea degree in electronic engineering and the Ph.D. degree in telecommunication engineering from the Università Politecnica delle Marche, Ancona, Italy, in 2001 and 2004, respectively.

From 2004 to 2006 he worked as Research Scientist (post-doc) in the Department of Electromagnetism and Bioengineering at Università Politecnica delle Marche. In 2006, he spent one year in Research and Development at medical and industrial optics industry (endoscope design and optical systems). He worked with National Nanotechnology Laboratory of CNR-INFM, Universitá del Salento. He is currently team leader in Robotics Lab. 1 of the Center of Bio-Molecular Nanotechnology of Italian Institute Technology (IIT), Arnesano, Lecce, Italy. His research interests are in the design and modeling of photonic band gap circuits, in the development of computer aided design (CAD) tools in the area of integrated optics, and in MEMS technology and systems.

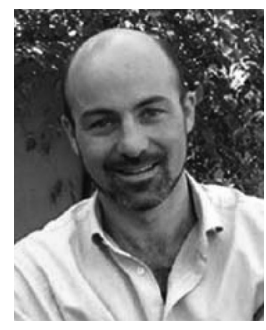

Roberto Cingolani was born in Milan, Italy, in December 1961. He received the Ph.D. degree in physics from the Scuola Normale Superiore di Pisa, Pisa, Italy.

From 1989 to 1991, he was a staff member at Max Planck Institute for Solid State Research-Stuttgart. During 1997, he was a Visiting Scientist at Tokyo University. Since 2000, he has been a Full Professor of experimental physics at the University of Salento, Lecce, Italy, where he has been the Director of the National Nanotechnology Laboratory of the Consiglio Nazionale delle Ricerche, Istituto Nazionale per la Fisica Della Materia, Disttretto Tecnologico Istituto Superiore di Formazione Interdisciplinare, since 2001, and the Scientific Director of the Italian Institute of Technology since 2004. His current research interests include nanotechnology and nanofabrication, semiconductor physics and devices, molecular organic nanostructures and devices, and bio-nanotechnologies.

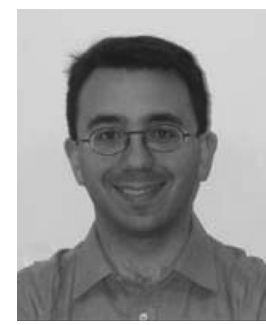

Massimo De Vittorio was born in Gallipoli (LE), Italy, in May 1967. He graduated in electronic engineering from the University of Pavia, Pavia, Italy, in 1992, and received the Master's degree in electronic engineering on semiconductor technology from the University of Lecce, Lecce, Italy, in 1995.

From 1996 to 2001, he was an Istituto Nazionale per la Fisica Della Materia (INFM) Senior Scientist Staff Member at the University of Lecce, where he was an Assistant Professor at the Engineering Faculty from 2001 to 2006. During 1999, he was a Visiting Scientist at the Center for Quantum Devices, North Western University. In 2000 he was an Invited Scientist at the Advanced Telecommunications Research Institute International, Adaptive Communications Research Laboratories, Japan. He is currently with the Nanodevice Division, National Nanotechnology Laboratory of the Consiglio Nazionale delle Ricerche, INFM, Disttretto Tecnologico Istituto Superiore di Formazione Interdisciplinare, Università del Salento, Lecce, Italy, where he has been an Associate Professor since 2006, and is currently a Lecturer of electronic and photonic devices, and nanotechnologies applied to electronic devices. His research interests include the design and fabrication of photonic and electronic devices based on either electronic or photonic lowdimensional structures.

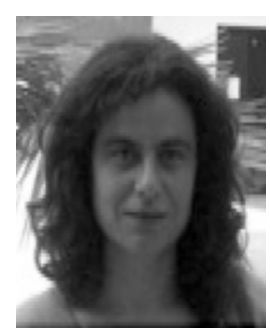

Adriana Passaseo is Researcher and Coordinator of the Inorganic Material Division, National Nanotechnology Laboratory from 2001. Its main research activities are on growth and characterization of III/V heterostructures, quantum nanostructures and III-Nitride heterostructures and related material and devices. She is author or coauthor of more than 160 international publications. 